Supporting Information

\title{
Donor-acceptor conjugated polymers with efficient thermally activated delayed fluorescence: random versus alternative polymerization
}

Xue Li, ${ }^{a, b, c}$ Jiancheng Rao, ${ }^{a, c}$ Liuqing Yang, ${ }^{a, b, c}$ Lei Zhao, ${ }^{a}$ Shumeng Wang, ${ }^{*, a}$ Hongkun Tian, ${ }^{* a, b}$ Junqiao Ding, ${ }^{*, a, b, c}$ Lixiang Wang $^{a, b}$

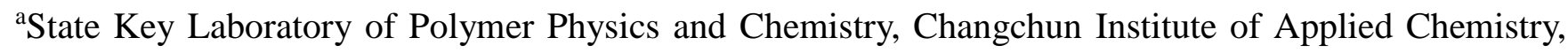
Chinese Academy of Sciences, Changchun 130022, P. R. China

${ }^{\mathrm{b} U n i v e r s i t y ~ o f ~ S c i e n c e ~ a n d ~ T e c h n o l o g y ~ o f ~ C h i n a, ~ H e f e i ~ 230026, ~ P . ~ R . ~ C h i n a ~}$

${ }^{\mathrm{c}}$ Key Laboratory of Medicinal Chemistry for Natural Resource, Ministry of Education; Yunnan Provincial Center for Research \& Development of Natural Products; School of Chemical Science and Technology, Yunnan University, Kunming 650091, P. R. China

E-mail: wangshumeng@ciac.ac.cn; hktian@ciac.ac.cn; dingjunqiao@ynu.edu.cn 


\section{Experimental section}

\section{Theoretical computation}

All quantum calculations were conducted by Gaussian 09 software package. ${ }^{1}$ The ground state geometries are optimized at B3LYP $/ 6-31 \mathrm{G}(\mathrm{d})$ level $^{3}$ by DFT. The excited $\mathrm{S}_{1}$ and $\mathrm{T}_{1}$ states simulations were carried out at the same level through TD-DFT methods using the optimized structures. The hole and electron analysis ${ }^{4}$ were performed by Multiwfn ${ }^{5}$ program and visualized by VMD ${ }^{6}$ software.

\section{Measurement and Characterization}

${ }^{1} \mathrm{H}$ and ${ }^{13} \mathrm{C}$ NMR spectra were recorded by Bruker Avance NMR spectrometers (500 MHz). Matrix-assisted laser desorption ionization/time-of-flight (MALDI-TOF) mass spectra were measured by AXIMA CFR MS apparatus (COMPACT). Elemental analysis (EA) was performed on elementary vario EL cube. Gel permeation chromatography (GPC) was measured on Waters 410 using polystyrene as the standard and THF as the eluent. Thermal gravimetric analysis (TGA) was performed with TA-TGA55 under nitrogen flow at a heating rate of $20^{\circ} \mathrm{C} / \mathrm{min}$. Differential scanning calorimetry (DSC) was performed on PerkinElmer-DSC 7 instrument under nitrogen flow at a heating rate of $10^{\circ} \mathrm{C} / \mathrm{min}$. UV-Visible absorption and photoluminescence (PL) spectra were collected with PerkinElmer Lambda 35 UV-vis spectrometer and PerkinElmer LS 50B spectrofluorometer, respectively. Transient PL spectra were detected on an Edinburgh fluorescence spectrometer (FLS-980). The absolute PL quantum yield (PLQY) was measured on HORIBA FL3C-111 combined with an integrating sphere under an excitation wavelength of $400 \mathrm{~nm}$. Cyclic voltammetry (CV) were performed on EG\&G 283 Princeton Applied Research potentiostat/galvanostat system using ferrocene/ferrocenium $\left(\mathrm{Fc} / \mathrm{Fc}^{+}\right)$as the reference and 
n- $\mathrm{Bu}_{4} \mathrm{NClO}_{4}$ as the supporting electrolyte. The HOMO and LUMO energy levels were calculated according to the equations: $\mathrm{HOMO}=-\mathrm{e}\left(\mathrm{E}_{\text {onset, ox }}+4.8 \mathrm{~V}\right), \mathrm{LUMO}=-\mathrm{e}\left(\mathrm{E}_{\text {onset, red }}+4.8 \mathrm{~V}\right)$, where $\mathrm{E}_{\text {onset, ox }}$ and $\mathrm{E}_{\text {onset, red }}$ are the onset values of the first oxidation and reduction waves, respectively.

\section{Device fabrication and testing}

The device configuration is ITO/PEDOT:PSS + PFI $(40 \mathrm{~nm}) / \mathrm{mCP}:$ polymer $(50 \mathrm{~nm}) / \mathrm{TSPO} 1(8 \mathrm{~nm})$ /TmPyPB $(42 \mathrm{~nm}) / \mathrm{LiF}(1 \mathrm{~nm}) / \mathrm{Al}(100 \mathrm{~nm})$. First, the pre-cleaned ITO-coated glass (15 $\Omega$ per square) were treated with ultraviolet-ozone for $40 \mathrm{~min}$. Second, the solution of PEDOT:PSS (Bayron P VP AI4083) and PFI with a volume ratio of 3:2 was spin-coated onto the substrate in air at a speed of 5000 rpm for $40 \mathrm{~s}$, annealed at $120{ }^{\circ} \mathrm{C}$ for $1 \mathrm{~h}$, and moved into a glove box filled with nitrogen. Third, the chlorobenzene solution of $\mathrm{mCP}$ and D-A conjugated polymer was spin-coated on the PEDOT: PSS + PFI layer at a speed of $1800 \mathrm{rpm}$ for $60 \mathrm{~s}$ to form the EML. Finally, other layers including $8 \mathrm{~nm}$ TSPO1, $42 \mathrm{~nm}$ TmPyPB, $1 \mathrm{~nm} \mathrm{LiF}$ and $100 \mathrm{~nm} \mathrm{Al}$ were successively evaporated in a vacuum chamber under a low base pressure $\left(<4 \times 10^{-4} \mathrm{~Pa}\right)$. The current density-voltage-luminance characteristics were measured by a Keithley source measurement unit (Keithley 2400 and Keithley 2000) together with a calibrated silicon photodiode. The EL spectra and CIE coordinates were tested on a CS2000 spectra colorimeter. EQE was calculated from the EL spectrum, luminance, and current density, assuming a Lambertian emission distribution. All the measurements were performed at room temperature under ambient atmosphere.

\section{Synthesis}


All raw materials were purchased by commercial channels and used directly without any purification unless pointed out. Solvents (DMF, THF, 1,4-dioxane and toluene) were dried by sodium and distilled under argon before use.

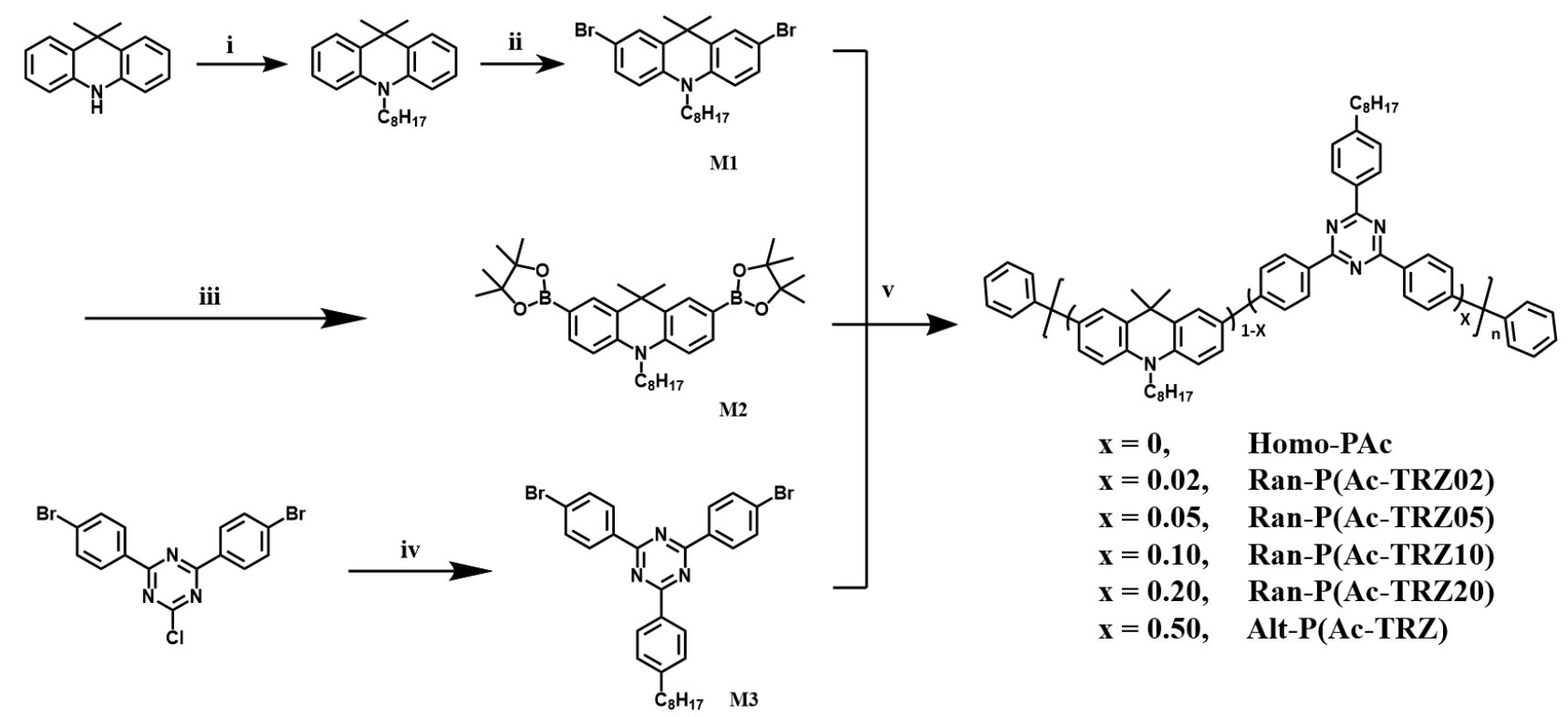

Scheme S1. Synthesis of the polymers. Regents and conditions: (i) 1-bromooctane, NaH, DMF, RT; (ii) NBS, DMF, $0{ }^{\circ} \mathrm{C}$ to RT; (iii) bis(pinacolato)diboron, $\mathrm{Pd}(\mathrm{dppf})_{2} \mathrm{Cl}_{2}, \mathrm{KOAc}$, Dioxane, $80{ }^{\circ} \mathrm{C}$; (iv) 1-bromo-4-octylbenzene, $\mathrm{Mg}, \mathrm{I}_{2}$, THF, $60{ }^{\circ} \mathrm{C}$; (v) $\mathrm{Pd}\left(\mathrm{pph}_{3}\right)_{4}$, Aliquat 336, $\mathrm{K}_{2} \mathrm{CO}_{3} / \mathrm{H}_{2} \mathrm{O}$, toluene.

9,9-dimethyl-10-octyl-9,10-dihydroacridine (1): 9,9-dimethyl-9,10-dihydroacridine (10 g, 47.8 mmol), 1-bromooctane (13.85 g, $71.7 \mathrm{mmol})$, sodium hydride (3.82 g, $95.6 \mathrm{mmol}, 40 \mathrm{wt} . \%)$ and DMF (200 mL) were stirred at room temperature under argon for $16 \mathrm{~h}$. The reaction mixture was dissolved by DCM $(500 \mathrm{~mL})$ and washed with saturated sodium chloride solution $(300 \mathrm{~mL})$. Then the organic layer was separated, dried over sodium sulfate, filtered and concentrated in vacuo. The residue was purified via column chromatography over silica gel using PE as eluent to give $\mathbf{1}$ as a colorless oil (12.77 g, yield 
83\%). ${ }^{1} \mathrm{H}$ NMR (500 MHz, d6-DMSO) $\delta 7.39(\mathrm{dd}, J=7.7,1.4 \mathrm{~Hz}, 2 \mathrm{H}), 7.21-7.15(\mathrm{~m}, 2 \mathrm{H}), 7.01(\mathrm{~d}, J=$ $7.7 \mathrm{~Hz}, 2 \mathrm{H}), 6.94-6.88(\mathrm{~m}, 2 \mathrm{H}), 3.99-3.86(\mathrm{~m}, 2 \mathrm{H}), 1.71(\mathrm{dt}, J=15.2,7.6 \mathrm{~Hz}, 2 \mathrm{H}), 1.44(\mathrm{~s}, 6 \mathrm{H}), 1.41$ $-1.19(\mathrm{~m}, 10 \mathrm{H}), 0.89-0.80(\mathrm{~m}, 3 \mathrm{H})$.

2,7-dibromo-9,9-dimethyl-10-octyl-9,10-dihydroacridine (M1): N-bromosuccinimide (14.85 g, 83.4 mmol) dissolved in DMF $(100 \mathrm{~mL})$ was added dropwise to a mixture of 9,9-dimethyl-10-octyl-9,10-dihydroacridine (1) $(12.77 \mathrm{~g}, 39.7 \mathrm{mmol})$ in $\mathrm{DMF}(100 \mathrm{~mL})$ at $0{ }^{\circ} \mathrm{C}$ and stirred for $3 \mathrm{~h}$ at room temperature. The reaction mixture was poured into $\mathrm{H}_{2} \mathrm{O}$, filtered and dissolved by EA. Then the organic layer was washed with saturated sodium chloride solution, dried over sodium sulfate, filtered and concentrated in vacuo. The residue was purified via column chromatography over silica gel using PE as eluent, and subsequently recrystallized from EtOH to give M1 as a white crystal (13 g, yield 68\%). ${ }^{1} \mathrm{H}$ NMR (500 MHz, $\left.\mathrm{CDCl}_{3}\right) \delta 7.46(\mathrm{~d}, J=2.3 \mathrm{~Hz}, 2 \mathrm{H}), 7.29(\mathrm{dd}, J=8.7,2.3 \mathrm{~Hz}, 2 \mathrm{H})$, $6.81(\mathrm{~d}, J=8.7 \mathrm{~Hz}, 2 \mathrm{H}), 3.87-3.80(\mathrm{~m}, 2 \mathrm{H}), 1.78(\mathrm{dt}, J=11.7,7.7 \mathrm{~Hz}, 2 \mathrm{H}), 1.49(\mathrm{~s}, 6 \mathrm{H}), 1.45-1.27$ (m, $10 \mathrm{H}), 0.90(\mathrm{t}, J=7.0 \mathrm{~Hz}, 3 \mathrm{H}) .{ }^{13} \mathrm{C} \mathrm{NMR}\left(126 \mathrm{MHz}, \mathrm{CDCl}_{3}\right) \delta 139.54,134.07,129.60,127.55$, $114.39,113.21,46.28,36.65,31.91,29.42,28.72,27.30,25.86,22.77,14.24$. MALDI-TOF (m/z) calcd for $\mathrm{C}_{23} \mathrm{H}_{27} \mathrm{Br}_{2} \mathrm{~N}[\mathrm{M}]^{+}$: 477.1; Found: 477.1.

9,9-dimethyl-10-octyl-2,7-bis(4,4,5,5-tetramethyl-1,3,2-dioxaborolan-2-yl)-9,10-dihydroacridine (M2): 2,7-Dibromo-9,9-dimethyl-10-octyl-9,10-dihydroacridine (M1) $\quad(5 \quad \mathrm{~g}, \quad 10.4$ mmol), bis(pinacolato)diboron (10.56 $\mathrm{g}, 41.6 \mathrm{mmol})$, potassium acetate $(3.06 \mathrm{~g}, 31.2 \mathrm{mmol})$ and [1,1'-bis(diphenylphosphino)ferrocene]dichloropalladium(II) $(0.46 \mathrm{~g}, 0.62 \mathrm{mmol})$ were deposited in a flask and degassed with argon. After the addition of 1,4-dioxane $(200 \mathrm{~mL})$, the reaction mixture was stirred for $4 \mathrm{~h}$ at $80{ }^{\circ} \mathrm{C}$, and then concentrated by rotary evaporation. The crude residue was purified via 
column chromatography over silica gel using $\mathrm{PE} / \mathrm{EA}(\mathrm{v} / \mathrm{v}=30: 1)$ as eluent, and subsequently recrystallized from EtOH to give $\mathbf{M} 2$ as a white solid (3 g, yield 50\%). ${ }^{1} \mathrm{H}$ NMR $\left(500 \mathrm{MHz}, \mathrm{CDCl}_{3}\right) \delta$ $7.90-7.78(\mathrm{~m}, 2 \mathrm{H}), 7.67(\mathrm{dd}, J=8.2,1.4 \mathrm{~Hz}, 2 \mathrm{H}), 6.94(\mathrm{~d}, J=8.3 \mathrm{~Hz}, 2 \mathrm{H}), 3.98-3.86(\mathrm{~m}, 2 \mathrm{H}), 1.82$ $(\mathrm{dt}, J=15.5,7.8 \mathrm{~Hz}, 2 \mathrm{H}), 1.70-1.52(\mathrm{~m}, 6 \mathrm{H}), 1.47(\mathrm{dd}, J=13.8,8.1 \mathrm{~Hz}, 2 \mathrm{H}), 1.44-1.26(\mathrm{~m}, 32 \mathrm{H})$, $0.90(\mathrm{t}, J=7.0 \mathrm{~Hz}, 3 \mathrm{H}) .{ }^{13} \mathrm{C} \mathrm{NMR}\left(126 \mathrm{MHz}, \mathrm{CDCl}_{3}\right) \delta 142.63,133.91,131.76,131.48,112.09,83.53$, $46.10,36.24,31.94,29.81,29.44,29.40,26.04,25.02,22.78,14.25$. MALDI-TOF (m/z) calcd for $\mathrm{C}_{35} \mathrm{H}_{54} \mathrm{~B}_{2} \mathrm{NO}_{4}[\mathrm{M}+\mathrm{H}]^{+}:$574.4; Found: 574.4 .

2,4-bis(4-bromophenyl)-6-(4-octylphenyl)-1,3,5-triazine (M3): Degassed 1-bromo-4-octylbenzene (5.39 g, $20 \mathrm{mmol})$ in THF (100 mL) was added slowly to $\mathrm{Mg}(0.58 \mathrm{~g}, 24 \mathrm{mmol})$ and a crystal of $\mathrm{I}_{2}$ under argon and stirred for $2 \mathrm{~h}$ at $60{ }^{\circ} \mathrm{C}$ to prepare the Grignard reagent (4-octylphenyl)magnesium bromide. Then the Grignard reagent was added to 2,4-bis(4-bromophenyl)-6-chloro-1,3,5-triazine (4.26 g, 10 mmol) in a flask under argon and stirred for $10 \mathrm{~h}$ at $60^{\circ} \mathrm{C}$. The reaction mixture was poured into $\mathrm{H}_{2} \mathrm{O}$, extracted by DCM, washed with saturated sodium chloride solution, and dried over sodium sulfate. After the removal of solvent, the residue was purified via column chromatography over silica gel using $\mathrm{PE} / \mathrm{EA}(\mathrm{v} / \mathrm{v}=20: 1)$ as eluent, and subsequently recrystallized from $\mathrm{PE} / \mathrm{DCM} / \mathrm{EtOH}$ mixed solvent to give $\mathrm{M3}$ as a white solid (1.9 g, yield 33\%). ${ }^{1} \mathrm{H} \mathrm{NMR}\left(500 \mathrm{MHz}, \mathrm{CDCl}_{3}\right) \delta 8.73-8.41(\mathrm{~m}, 6 \mathrm{H}), 7.81-$ $7.53(\mathrm{~m}, 4 \mathrm{H}), 7.49-7.17(\mathrm{~m}, 2 \mathrm{H}), 2.84-2.56(\mathrm{~m}, 2 \mathrm{H}), 1.78-1.54(\mathrm{~m}, 2 \mathrm{H}), 1.46-1.18(\mathrm{~m}, 10 \mathrm{H}), 1.00$ $-0.80(\mathrm{~m}, 3 \mathrm{H}) .{ }^{13} \mathrm{C} \mathrm{NMR}\left(126 \mathrm{MHz}, \mathrm{CDCl}_{3}\right) \delta 171.96,170.89,148.64,135.21,133.41,132.02,130.56$, $129.15,128.96,127.65,36.27,32.03,31.39,29.63,29.47,29.43,22.82,14.26$. MALDI-TOF (m/z) calcd for $\mathrm{C}_{29} \mathrm{H}_{28} \mathrm{Br}_{2} \mathrm{~N}_{3}[\mathrm{M}+\mathrm{H}]^{+}$: 578.1; Found: 578.1 . 
General synthetic procedure for the polymers: The polymers were synthesized according to a general procedure of Pd-catalyzed Suzuki polycondensation. Bromine monomer $(\mathbf{M 1}+\mathbf{M 3}=0.5 \mathrm{mmol})$, 9,9-dimethyl-10-octyl-2,7-bis(4,4,5,5-tetramethyl-1,3,2-dioxaborolan-2-yl)-9,10-dihydroacridine (M2) (286.7 $\mathrm{mg}, 0.5 \mathrm{mmol})$, two drop of Aliquat $336(\sim 50 \mathrm{mg})$ and tetrakis(triphenylphosphine)palladium $(5.8 \mathrm{mg}$ ) were degassed for $30 \mathrm{~min}$ in a flask. The degassed toluene was added to the mixture and stirred under argon. Potassium carbonate solution $\left(5 \mathrm{~mL}, 2 \mathrm{~mol} / \mathrm{L}\right.$ in $\mathrm{H}_{2} \mathrm{O}$ ) was added when the reaction temperature was heated to $80{ }^{\circ} \mathrm{C}$, which was further raised to $95{ }^{\circ} \mathrm{C}$. When the viscosity of the mixture was enough, phenylboronic acid (50 $\mathrm{mg}$ in $5 \mathrm{~mL}$ toluene) and bromobenzene $(0.5 \mathrm{~mL})$ were added to end-cap the polymers in sequence. Finally, sodium diethyldithiocarbamate trihydrate $(10 \mathrm{~mL}, 1 \mathrm{~g} / \mathrm{mL}$ in $\mathrm{H}_{2} \mathrm{O}$ ) was added and stirred at $80{ }^{\circ} \mathrm{C}$ for $12 \mathrm{~h}$. After cooling to room temperature, the reaction mixture was poured into toluene, washed by saturated sodium chloride solution, dried over sodium sulfate, purified via column chromatography over silica gel using toluene as eluent and concentrated via rotary evaporation. The polymers were precipitated from $\mathrm{MeOH}(50 \mathrm{~mL})$, filtered, purified by Soxlet extraction with acetone at $80^{\circ} \mathrm{C}$ for $24 \mathrm{~h}$ and dried via vacuum to produce the final product.

Homo-PAc (white solid, $100 \mathrm{mg}$, yield 31\%): M1 (239.7 mg, $0.5 \mathrm{mmol})$ and $\mathbf{M 2}(286.7 \mathrm{mg}, 0.5$ mmol) were used in polymerization. ${ }^{1} \mathrm{H}$ NMR $\left(500 \mathrm{MHz}, \mathrm{CDCl}_{3}\right) \delta 7.77-7.60(\mathrm{~m}, 2 \mathrm{H}), 7.47(\mathrm{t}, J=7.0$ $\mathrm{Hz}, 2 \mathrm{H}), 7.04$ (m, 2H), 4.00 (s, 2H), 1.93 (s, 2H), $1.74-1.67$ (m, 6H), $1.59-1.30$ (m, 10H), 0.93 (dd, $J$ $=7.2,3.6 \mathrm{~Hz}, 3 \mathrm{H}) .{ }^{13} \mathrm{C} \mathrm{NMR}\left(126 \mathrm{MHz}, \mathrm{CDCl}_{3}\right) \delta 141.57,139.24,133.74,132.48,128.86,126.75$, $126.53,125.02,123.15,112.99,46.31,36.65,31.99,29.57,29.51,27.46,26.16,22.82,14.28$. Elemental analysis calcd (\%) for $\left(\mathrm{C}_{23} \mathrm{H}_{29} \mathrm{~N}\right)_{\mathrm{n}}$ : C, 86.47; H, 9.15; N, 4.38. Found: C, 86.29; H, 8.40; N, 4.13. 
Ran-P(Ac-TRZ02) (yellow solid, 80 mg, yield 25\%): M1 (230.1 mg, 0.48 mmol), M2 (286.7 mg, 0.5 $\mathrm{mmol})$ and $\mathbf{M 3}(11.6 \mathrm{mg}, 0.02 \mathrm{mmol})$ were used in polymerization. ${ }^{1} \mathrm{H} \mathrm{NMR}\left(500 \mathrm{MHz}, \mathrm{CDCl}_{3}\right) \delta 8.87$ $(\mathrm{d}, J=8.2 \mathrm{~Hz}, 0.111 \mathrm{H}), 8.74(\mathrm{~d}, J=8.0 \mathrm{~Hz}, 0.056 \mathrm{H}), 7.87-7.82(\mathrm{~m}, 0.167 \mathrm{H}), 7.74-7.60(\mathrm{~m}, 2.004 \mathrm{H})$, $7.45(\mathrm{dd}, J=16.0,8.0 \mathrm{~Hz}, 1.999 \mathrm{H}), 7.20-6.90(\mathrm{~m}, 2.000 \mathrm{H}), 4.01(\mathrm{~s}, 2.001 \mathrm{H}), 2.76(\mathrm{~s}, 0.055 \mathrm{H}), 1.92(\mathrm{~s}$, $2.002 \mathrm{H}), 1.77-1.63(\mathrm{~m}, 6.003 \mathrm{H}), 1.58-1.29(\mathrm{~m}, 10.334 \mathrm{H}), 0.92(\mathrm{t}, J=6.6 \mathrm{~Hz}, 3.084 \mathrm{H}) .{ }^{13} \mathrm{C} \mathrm{NMR}$ $\left(126 \mathrm{MHz}, \mathrm{CDCl}_{3}\right) \delta 141.56,139.23,133.82,132.43,129.66,128.86,126.75,126.53,125.48,125.04$, $123.63,123.13,113.00,46.33,36.65,31.99,29.57,29.52,27.46,26.18,22.82,14.28$. Elemental analysis calcd (\%) for $\left(\mathrm{C}_{23.12} \mathrm{H}_{29} \mathrm{~N}_{1.04}\right)_{n}: \mathrm{C}, 86.37 ; \mathrm{H}, 9.09 ; \mathrm{N}, 4.53$. Found: $\mathrm{C}, 86.13 ; \mathrm{H}, 8.65 ; \mathrm{N}, 4.24$.

Ran-P(Ac-TRZ05) (yellow solid, $122 \mathrm{mg}$, yield 37\%): M1 (215.7 mg, $0.45 \mathrm{mmol}$ ), M2 (286.7 mg, $0.5 \mathrm{mmol})$ and $\mathbf{M 3}(29.0 \mathrm{mg}, 0.05 \mathrm{mmol})$ were used in polymerization. ${ }^{1} \mathrm{H} \mathrm{NMR}\left(500 \mathrm{MHz}, \mathrm{CDCl}_{3}\right) \delta$ $8.88(\mathrm{~d}, J=6.5 \mathrm{~Hz}, 0.222 \mathrm{H}), 8.75(\mathrm{~d}, J=6.4 \mathrm{~Hz}, 0.111 \mathrm{H}), 7.88-7.83(\mathrm{~m}, 0.333 \mathrm{H}), 7.74-7.62(\mathrm{~m}$, $2.000 \mathrm{H}), 7.46(\mathrm{dd}, J=13.8,6.5 \mathrm{~Hz}, 2.000 \mathrm{H}), 7.23-6.88(\mathrm{~m}, 2.000 \mathrm{H}), 4.03(\mathrm{~s}, 2.000 \mathrm{H}), 2.76(\mathrm{~d}, J=5.6$ $\mathrm{Hz}, 0.111 \mathrm{H}), 1.94(\mathrm{~s}, 2.001 \mathrm{H}), 1.76-1.66(\mathrm{~m}, 6.006 \mathrm{H}), 1.58-1.30(\mathrm{~m}, 10.667 \mathrm{H}), 0.98-0.90(\mathrm{~m}$, 3.167H). ${ }^{13} \mathrm{C}$ NMR $\left(126 \mathrm{MHz}, \mathrm{CDCl}_{3}\right) \delta 171.68,171.45,148.14,145.34,141.60,139.24,133.67$, $132.42,129.63,128.85,126.74,126.65,125.03,123.72,123.14,113.08,112.97,46.32,36.70,31.99$, $31.47,29.57,29.52,27.46,26.18,22.82,14.28$. Elemental analysis calcd $(\%)$ for $\left(\mathrm{C}_{23.3} \mathrm{H}_{29} \mathrm{~N}_{1.1}\right)_{\mathrm{n}}$ : $\mathrm{C}$, 86.24; H, 9.01; N, 4.75. Found: C, 86.17; H, 8.65; N, 4.48.

Ran-P(Ac-TRZ10) (yellow solid, $82 \mathrm{mg}$, yield 25\%): M1 (191.7 mg, $0.4 \mathrm{mmol}$ ), M2 (286.7 mg, 0.5 mmol) and $\mathbf{M 3}(57.9 \mathrm{mg}, 0.1 \mathrm{mmol})$ were used in polymerization. ${ }^{1} \mathrm{H}$ NMR $\left(500 \mathrm{MHz}, \mathrm{CDCl}_{3}\right) \delta 8.88$ $(\mathrm{d}, J=7.8 \mathrm{~Hz}, 0.478 \mathrm{H}), 8.74(\mathrm{~d}, J=7.8 \mathrm{~Hz}, 0.242 \mathrm{H}), 7.85(\mathrm{~d}, J=8.2 \mathrm{~Hz}, 0.716 \mathrm{H}), 7.72-7.61(\mathrm{~m}$, 2.012H), $7.46(\mathrm{dd}, J=17.7,8.4 \mathrm{~Hz}, 2.003 \mathrm{H}), 7.20-6.99(\mathrm{~m}, 2.000 \mathrm{H}), 4.03(\mathrm{~s}, 2.011 \mathrm{H}), 2.76(\mathrm{~s}$, 
$0.235 \mathrm{H}), 1.93(\mathrm{~s}, 2.006 \mathrm{H}), 1.78-1.64(\mathrm{~m}, 6.004 \mathrm{H}), 1.58-1.31(\mathrm{~m}, 11.415 \mathrm{H}), 0.93(\mathrm{t}, J=6.5 \mathrm{~Hz}$ 3.361H). $\left.{ }^{13} \mathrm{C} \mathrm{NMR} \mathrm{(126} \mathrm{MHz,} \mathrm{CDCl}_{3}\right) \delta 171.66,171.43,148.18,145.36,141.56,140.35,139.23$ $134.52,134.10,133.24,132.62,132.43,129.66,129.17,128.93,128.86,126.75,126.65,126.53,125.77$ $125.47,125.08,123.74,123.17,113.26,113.10,113.00,46.35,36.70,36.29,32.05,31.99,31.47,29.65$, 29.57, 29.52, 29.44, 27.46, 26.19, 22.82, 14.28. Elemental analysis calcd (\%) for $\left(\mathrm{C}_{23.6} \mathrm{H}_{29} \mathrm{~N}_{1.2}\right)_{\mathrm{n}}$ : C, 86.03; H, 8.87; N, 5.10. Found: C, 85.48; H, 8.55; N, 4.81.

Ran-P(Ac-TRZ20) (yellow solid, 127 mg, yield 37\%): M1 (143.8 mg, 0.3 mmol), M2 (286.7 mg, 0.5 mmol) and $\mathbf{M 3}(115.9 \mathrm{mg}, 0.2 \mathrm{mmol})$ were used in polymerization. ${ }^{1} \mathrm{H} \mathrm{NMR}\left(500 \mathrm{MHz}, \mathrm{CDCl}_{3}\right) \delta 8.88$ $(\mathrm{d}, J=6.0 \mathrm{~Hz}, 1.000 \mathrm{H}), 8.75(\mathrm{~d}, J=7.5 \mathrm{~Hz}, 0.499 \mathrm{H}), 7.85(\mathrm{~d}, J=7.6 \mathrm{~Hz}, 1.500 \mathrm{H}), 7.73-7.61(\mathrm{~m}$, $1.999 \mathrm{H}), 7.53-7.40(\mathrm{~m}, 1.999 \mathrm{H}), 7.10(\mathrm{dd}, J=15.2,7.0 \mathrm{~Hz}, 2.000 \mathrm{H}), 4.03(\mathrm{~s}, 1.999 \mathrm{H}), 2.77$ (s, $0.500 \mathrm{H}), 1.95(\mathrm{~s}, 2.000 \mathrm{H}), 1.79-1.67(\mathrm{~m}, 5.997 \mathrm{H}), 1.61-1.28(\mathrm{~m}, 12.993 \mathrm{H}), 1.00-0.87(\mathrm{~m}, 3.748 \mathrm{H})$. ${ }^{13} \mathrm{C}$ NMR $\left(126 \mathrm{MHz}, \mathrm{CDCl}_{3}\right) \delta 171.66,171.43,148.13,145.33,140.34,139.06,134.67,134.13,132.61$, $132.42,129.64,129.15,128.92,126.63,125.84,125.07,123.78,123.18,113.09,46.34,36.70,36.29$, $32.05,31.99,31.46,29.65,29.57,29.52,29.44,27.46,26.21,22.83,14.28$. Elemental analysis calcd (\%) for $\left(\mathrm{C}_{24.2} \mathrm{H}_{29} \mathrm{~N}_{1.4}\right)_{\mathrm{n}}: \mathrm{C}, 85.61 ; \mathrm{H}, 8.61 ; \mathrm{N}, 5.78$. Found: $\mathrm{C}, 85.30 ; \mathrm{H}, 8.38 ; \mathrm{N}, 5.61$.

Alt-P(Ac-TRZ) (yellow solid, $200 \mathrm{mg}$, yield 54\%): M2 (286.7 mg, $0.5 \mathrm{mmol}$ ) and M3 (289.7 mg, 0.5 mmol) were used in polymerization. ${ }^{1} \mathrm{H}$ NMR $\left(500 \mathrm{MHz}, \mathrm{CDCl}_{3}\right) \delta 8.84(\mathrm{~d}, J=7.1 \mathrm{~Hz}, 4 \mathrm{H}), 8.71(\mathrm{~d}, J=$ $7.3 \mathrm{~Hz}, 2 \mathrm{H}), 7.82(\mathrm{~d}, J=8.2 \mathrm{~Hz}, 6 \mathrm{H}), 7.60(\mathrm{~d}, J=7.2 \mathrm{~Hz}, 2 \mathrm{H}), 7.39(\mathrm{~d}, J=7.0 \mathrm{~Hz}, 2 \mathrm{H}), 7.08(\mathrm{~d}, J=8.3$ $\mathrm{Hz}, 2 \mathrm{H}), 4.00(\mathrm{~s}, 2 \mathrm{H}), 2.74(\mathrm{~s}, 2 \mathrm{H}), 1.93(\mathrm{~s}, 2 \mathrm{H}), 1.72(\mathrm{~d}, J=19.6 \mathrm{~Hz}, 6 \mathrm{H}), 1.55-1.24(\mathrm{~m}, 22 \mathrm{H}), 0.91$ $(\mathrm{m}, 6 \mathrm{H}) .{ }^{13} \mathrm{C}$ NMR $\left(126 \mathrm{MHz}, \mathrm{CDCl}_{3}\right) \delta 171.61,171.36,148.11,145.13,140.31,134.62,134.10,132.58$, $129.64,129.15,128.89,126.59,125.81,123.74,113.24,46.36,36.72,36.28,32.05,31.45,29.86,29.81$, 
29.66, 29.56, 29.53, 29.51, 29.45, 27.43, 26.21, 22.84, 14.27. Elemental analysis calcd (\%) for $\left(\mathrm{C}_{52} \mathrm{H}_{58} \mathrm{~N}_{4}\right)_{\mathrm{n}}: \mathrm{C}, 84.51 ; \mathrm{H}, 7.91 ; \mathrm{N}, 7.58$. Found: C, 84.20; H, 7.92; N, 7.24.

\section{References:}

1. Frisch, M. J.; Trucks, G. W.; Schlegel, H. B.; Scuseria, G. E.; Robb, M. A.; Cheeseman, J. R.;

Scalmani, G.; Barone, V.; Mennucci, B.; Petersson, G. A.; Nakatsuji, H.; Caricato, M.;Li, X.;

Hratchian, H. P.; Izmaylov, A. F.; Bloino, J.; Zheng, G.; Sonnenberg, J. L.; Hada, M.; Ehara, M.;

Toyota, K.; Fukuda, R.; Hasegawa, J.; Ishida, M.; Nakajima, T.; Honda, Y.; Kitao, O.; Nakai, H.;

Vreven, T.; Montgomery, Jr., J. A.; Peralta, J. E.; Ogliaro, F.; Bearpark, M.; Heyd, J. J.; Brothers, E.;

Kudin, K. N.; Staroverov, V. N.; Kobayashi, R.; Normand, J.; Raghavachari, K.; Rendell, A.; Burant,

J. C.; Iyengar, S. S.; Tomasi, J.; Cossi, M.; Rega, N.; Millam, N. J.; Klene, M.; Knox, J. E.; Cross, J.

B.; Bakken, V.; Adamo, C.; Jaramillo, J.; Gomperts, R.; Stratmann, R. E.; Yazyev, O.; Austin, A. J.;

Cammi, R.; Pomelli, C.; Ochterski, J. W.; Martin, R. L.; Morokuma, K.; Zakrzewski, V. G.; Voth, G.

A.; Salvador, P.; Dannenberg, J. J.; Dapprich, S.; Daniels, A. D.; Farkas, Ö.; Foresman, J. B.; Ortiz, J.

V.; Cioslowski, J.; Fox, D. J. Gaussian, Inc., Wallingford CT, 2009.

2. Becke, A. D. Density-functional thermochemistry. III. The role of exact exchangel. J. Chem. Phys.

1993, $98,5648$.

3. Ditchfield, R.; Hehre, W. J.; Pople, J. A. Self-consistent molecular-orbital methods. IX. An extended Gaussian-type basis for molecular-orbital studies of organic molecules. J. Chem. Phys. 1971, 54, 724-728.

4. Lu, T. Multiwfn Manual, version 3.7(dev), Section 3.21.1, available at http://sobereva.com/multiwfn 
(accessed Mar. 23, 2020).

5. Lu, T.; Chen, F. Multiwfn: a multifunctional wavefunction analyzer. J. Comput. Chem. 2012, 33, 580-592.

6. William, H., Andrew, D., Klaus, S., VMD: Visual Molecular Dynamics. J. Mol. Graphics, 1996, 14, 33-38. 

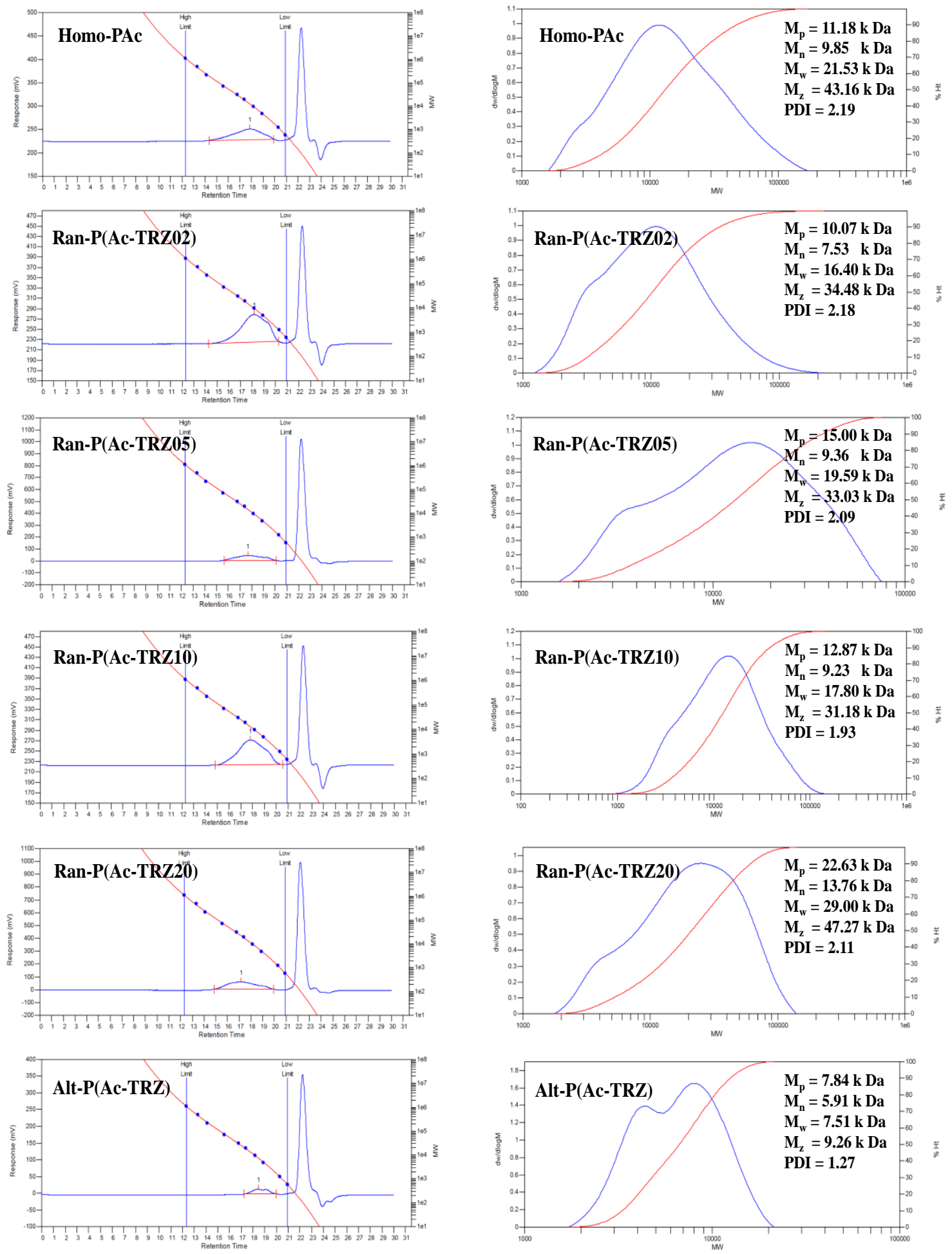

Figure S1. GPC traces of Homo-PAc, Ran-P(Ac-TRZ02) Ran-P(Ac-TRZ20) and Alt-P(Ac-TRZ). We note that most of these polymers show a bimodal GPC trace, which could be tentatively ascribed to their moderate solubility. 

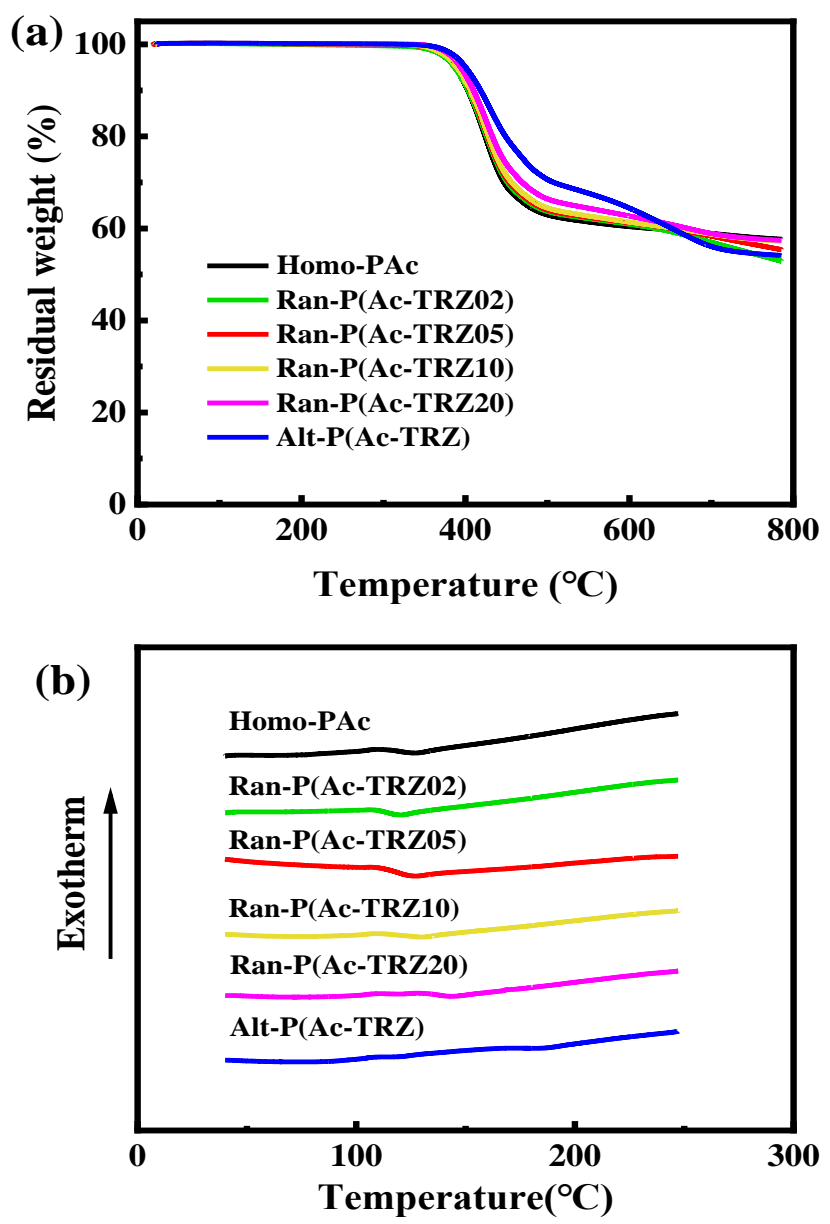

Figure S2. TGA (a) and DSC profiles (b) for Homo-PAc, Ran-P(Ac-TRZ02) Ran-P(Ac-TRZ20) and Alt-P(Ac-TRZ). 


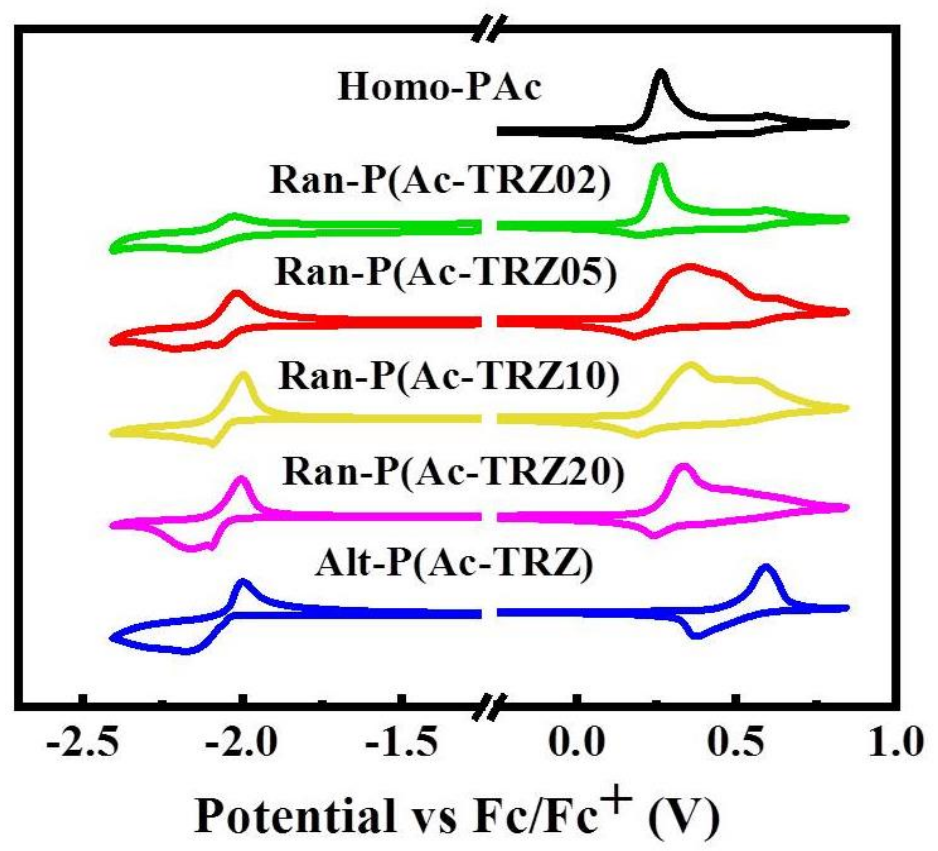

Figure S3. CV curves for Homo-PAc, Ran-P(Ac-TRZ02) Ran-P(Ac-TRZ20) and Alt-P(Ac-TRZ). Here $N, N$-dimethylformamide (DMF) is used as the solvent for anodic and cathodic sweeping. 


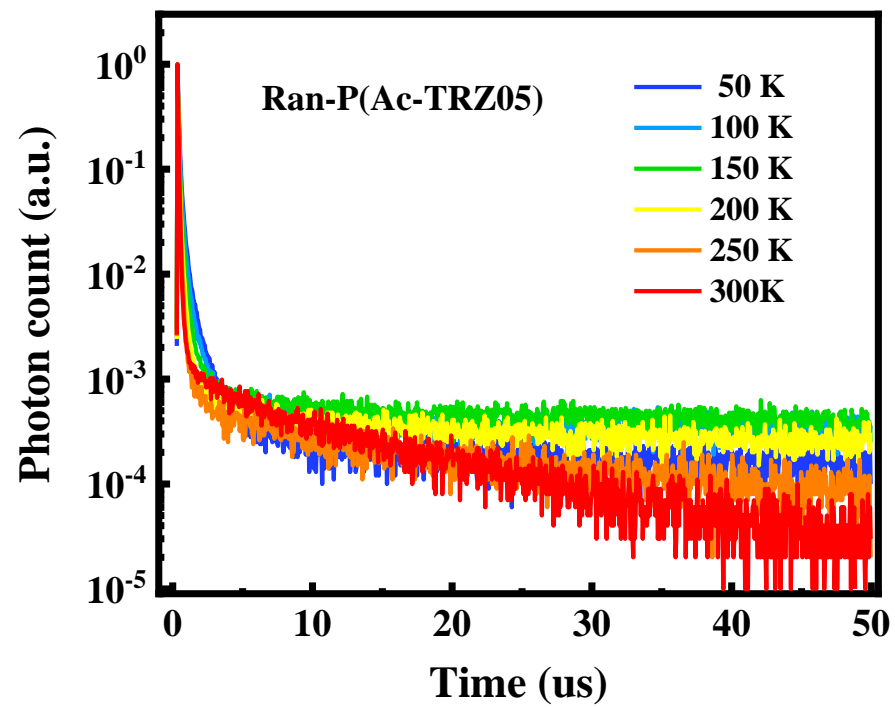

Figure S4. Temperature-dependent transient PL spectra in neat films taking Ran-P(Ac-TRZ05) as an example. 

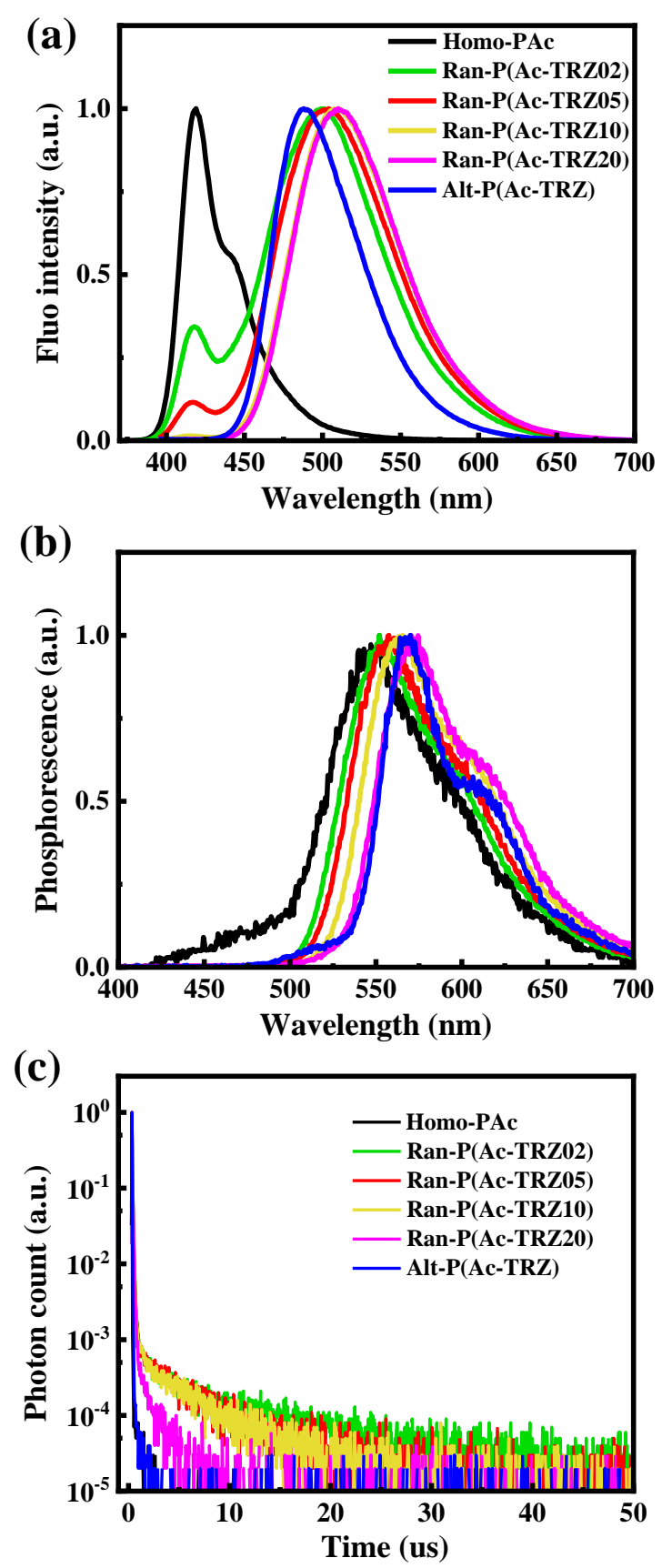

Figure S5. Photophysical properties of Ran-P(Ac-TRZ02) Ran-P(Ac-TRZ20) in 5 wt.\% doped mCP films compared with those of Homo-PAc and Alt-P(Ac-TRZ): (a) fluorescence; (b) phosphorescence measured at $77 \mathrm{~K}$; (c) transient PL spectra. 


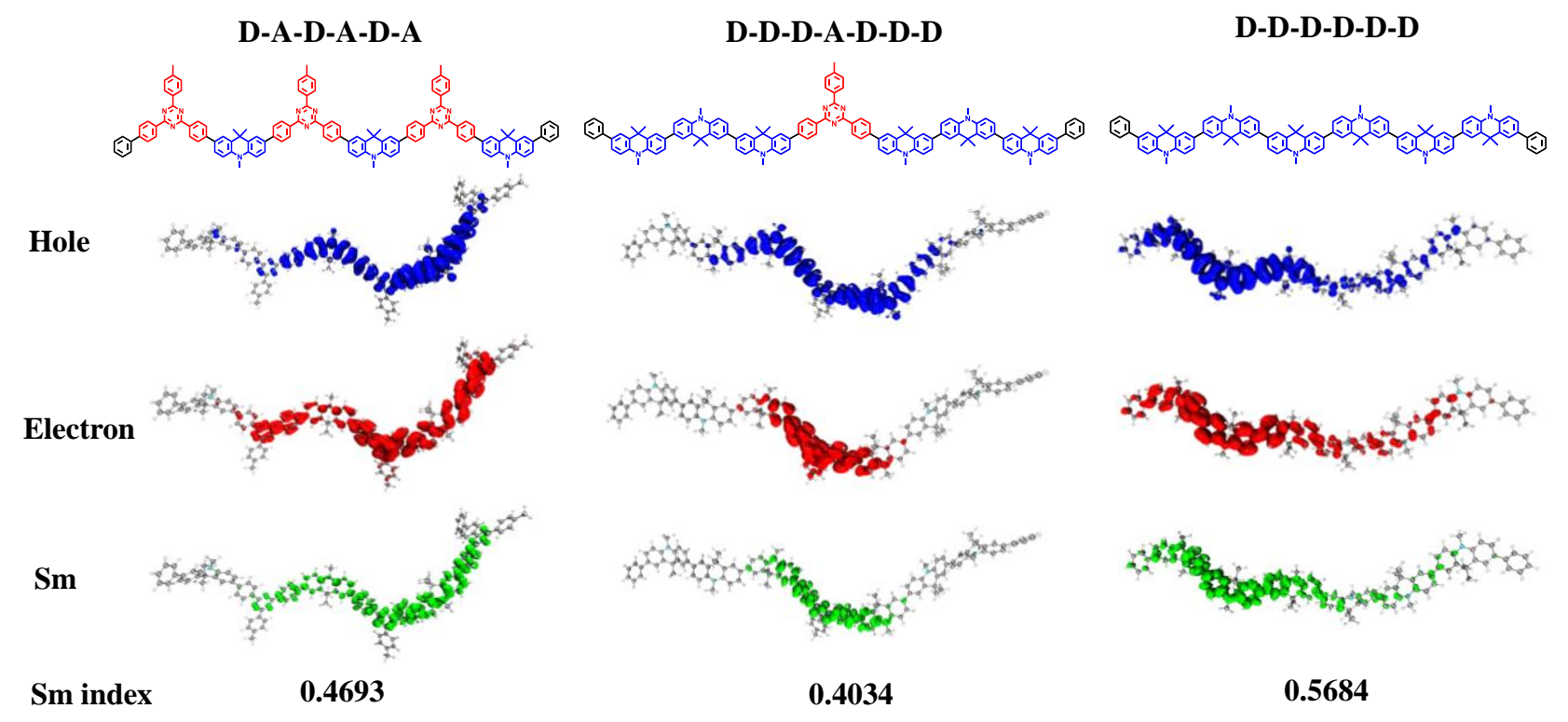

Figure S6. Molecular structures of chain segments involved in alternative and random polymers, and their hole/electron distributions of the $T_{1}$ states. $S_{m}$ is the overlap function between hole and electron distributions, quantified by the $\mathrm{S}_{\mathrm{m}}$ index ranging from 0 (non-overlap) and 1 (complete overlap). 


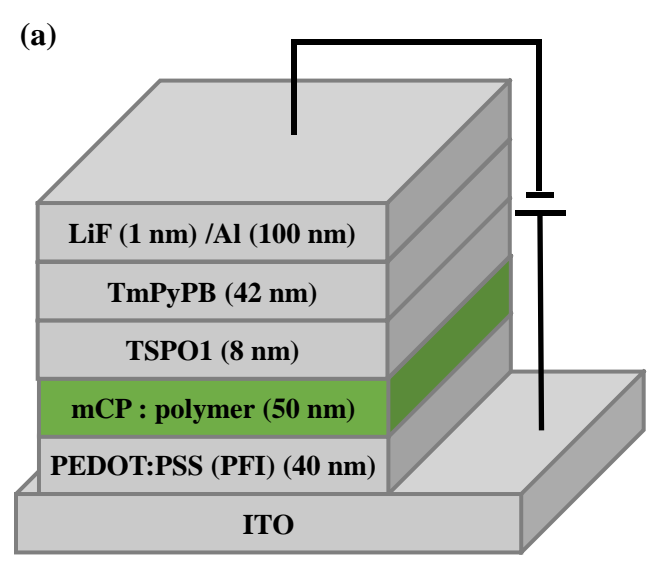

(b)

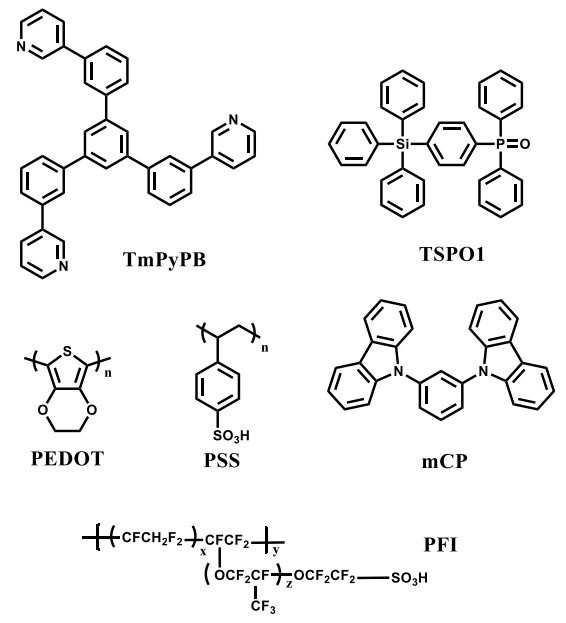

Figure S7. Device configuration (a) and the molecular structures of used materials (b). 

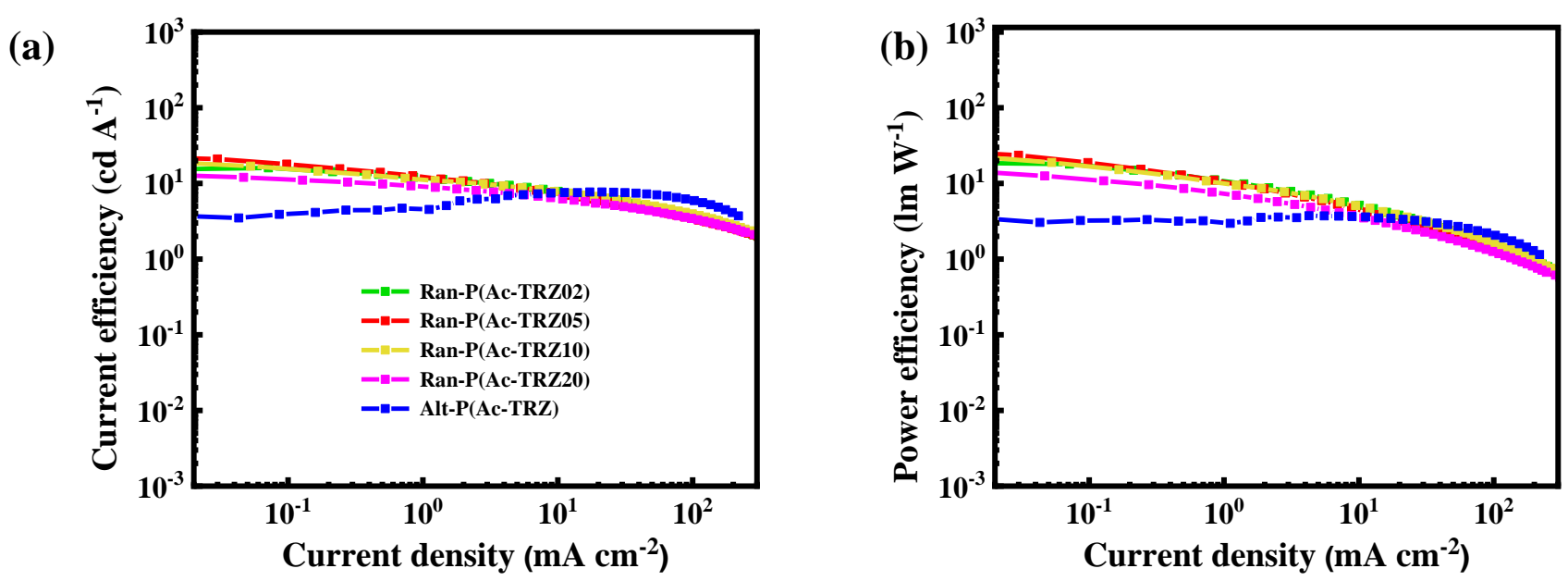

Figure S8. Current efficiency (a) and power efficiency (b) as a function of current density for Ran-P(Ac-TRZ02) Ran-P(Ac-TRZ20) compared with Alt-P(Ac-TRZ). 


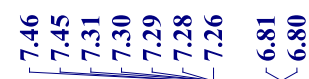

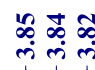

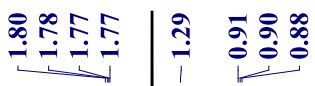
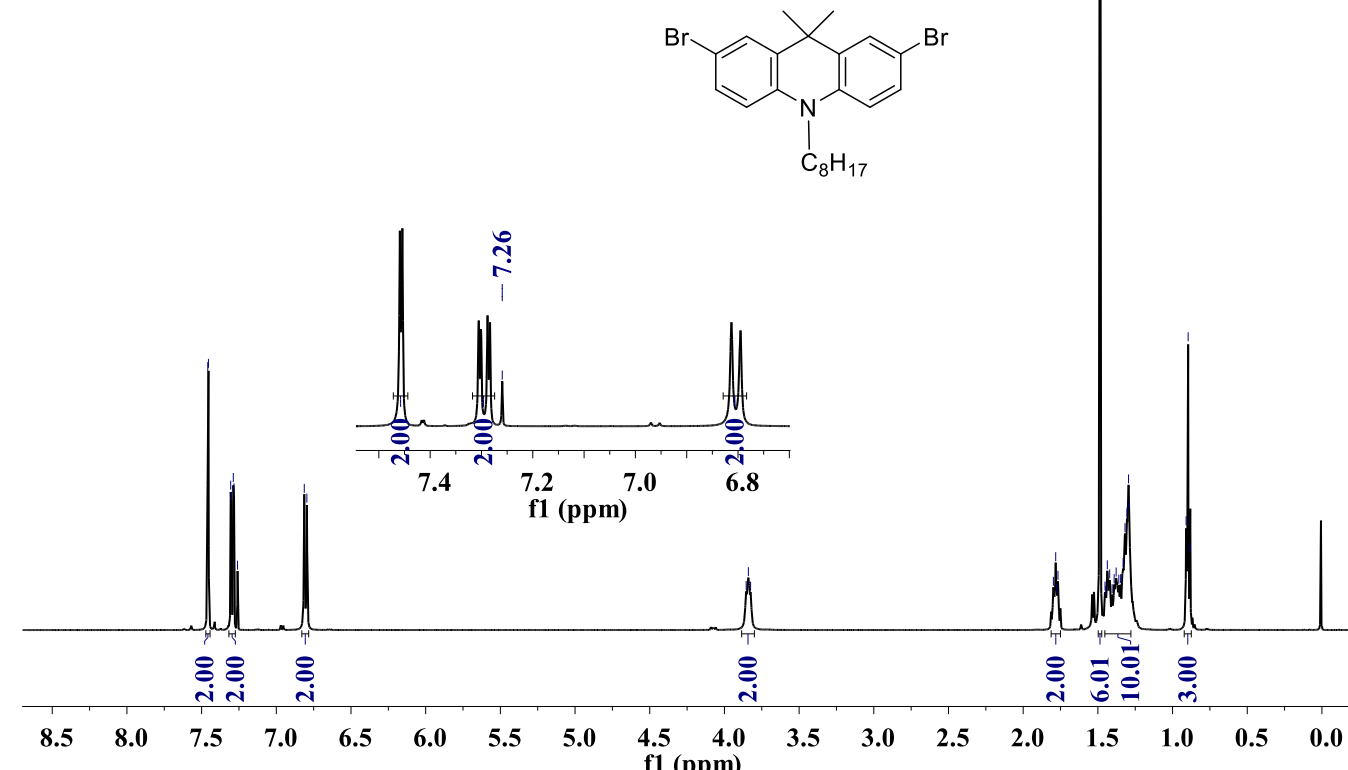

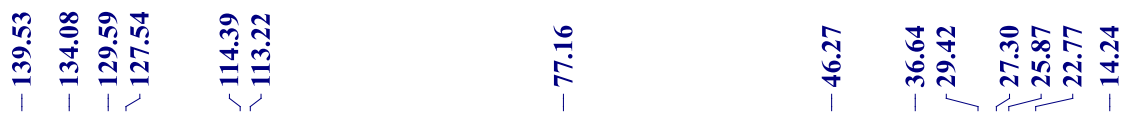

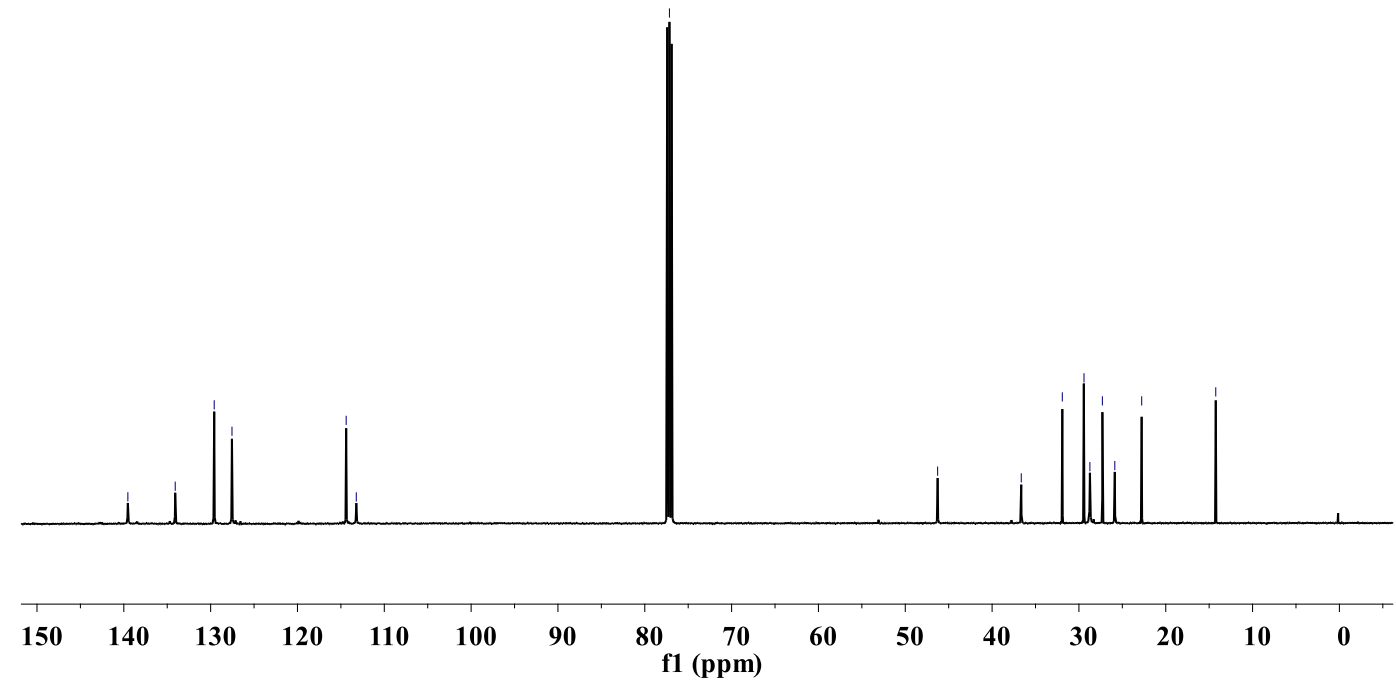

Figure S9. ${ }^{1} \mathrm{H}$ and ${ }^{13} \mathrm{C}$ NMR spectra of $\mathbf{M 1}$. 

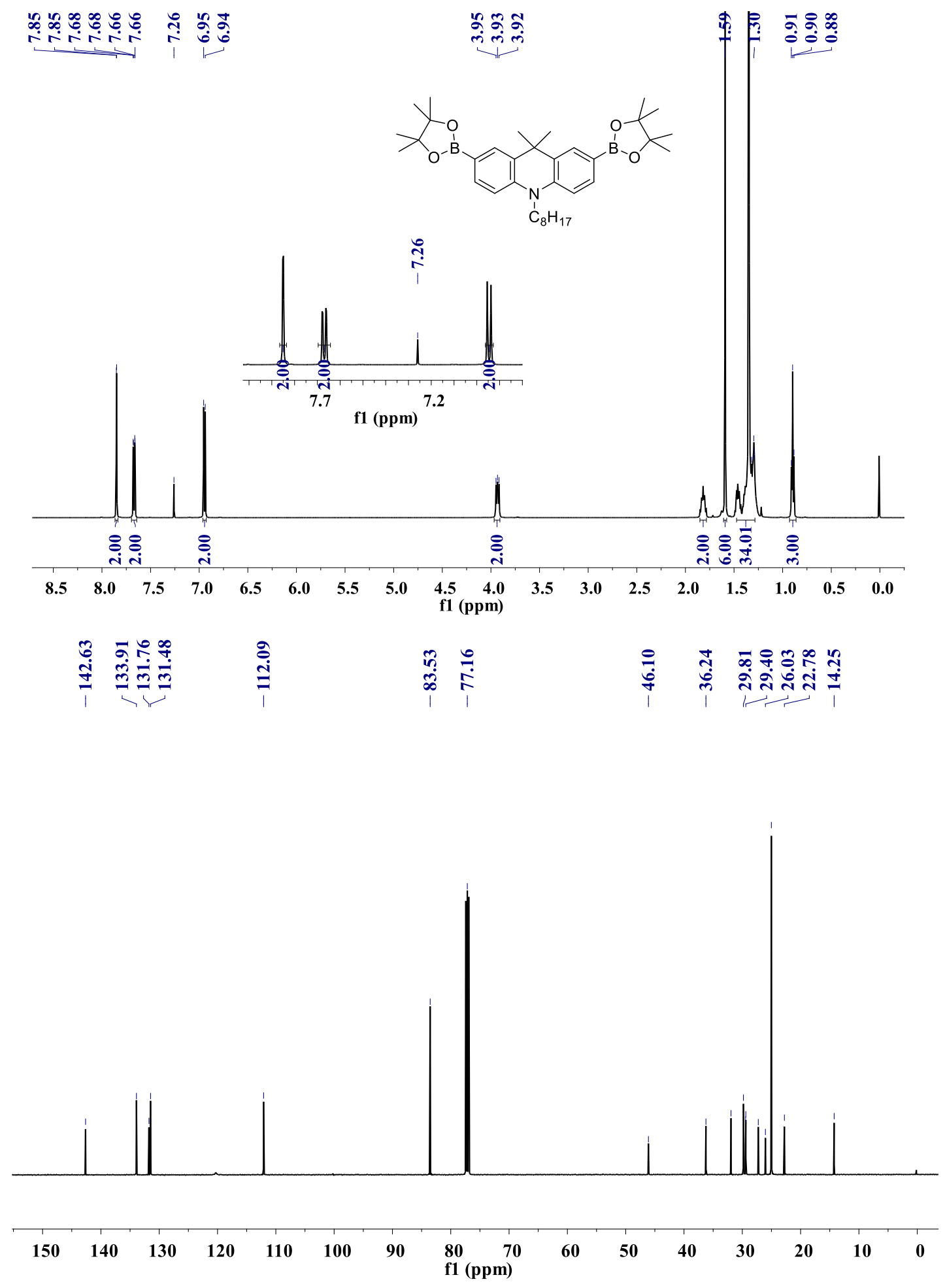

Figure S10. ${ }^{1} \mathrm{H}$ and ${ }^{13} \mathrm{C}$ NMR spectra of $\mathbf{M} 2$. 


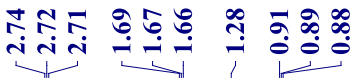
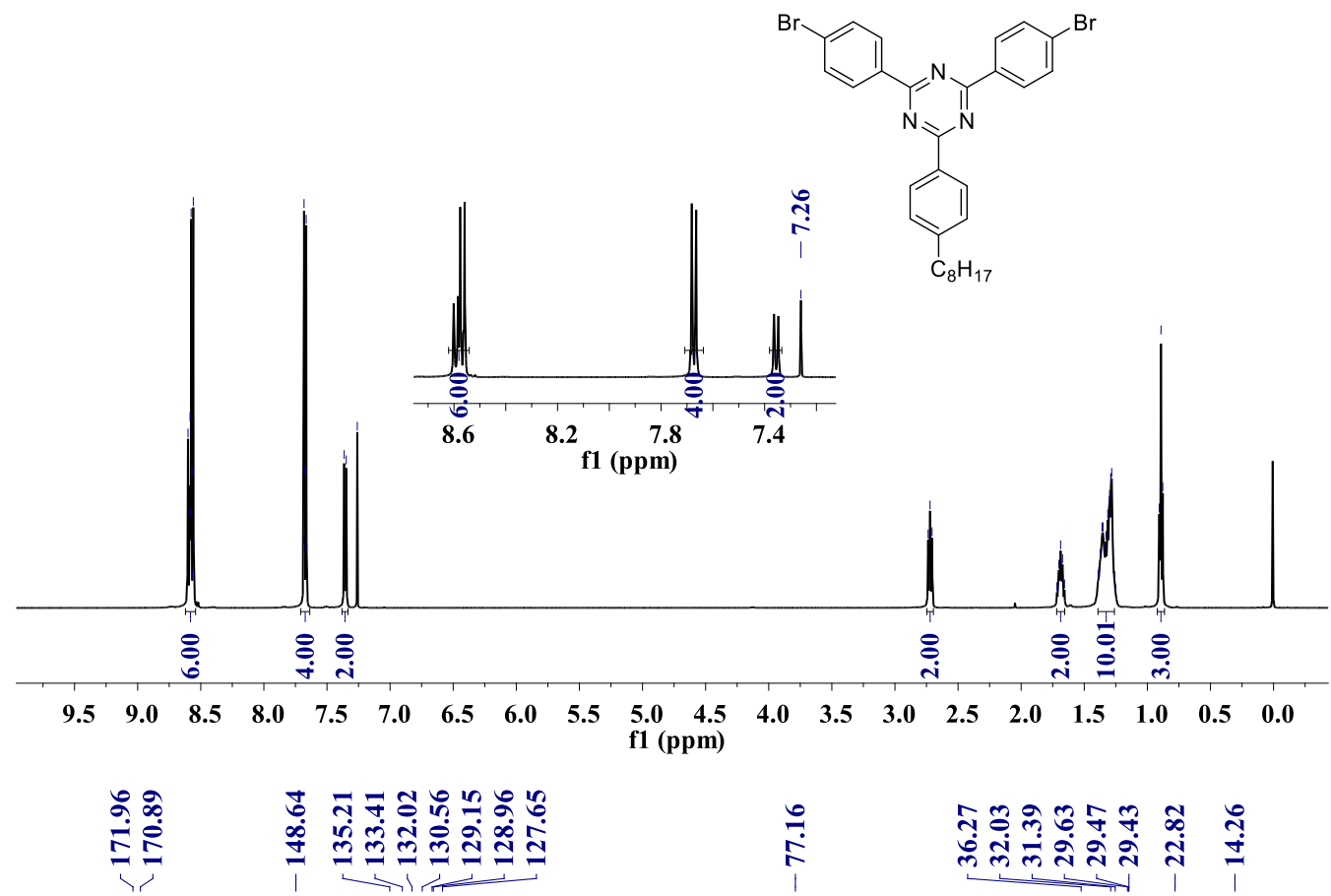

군

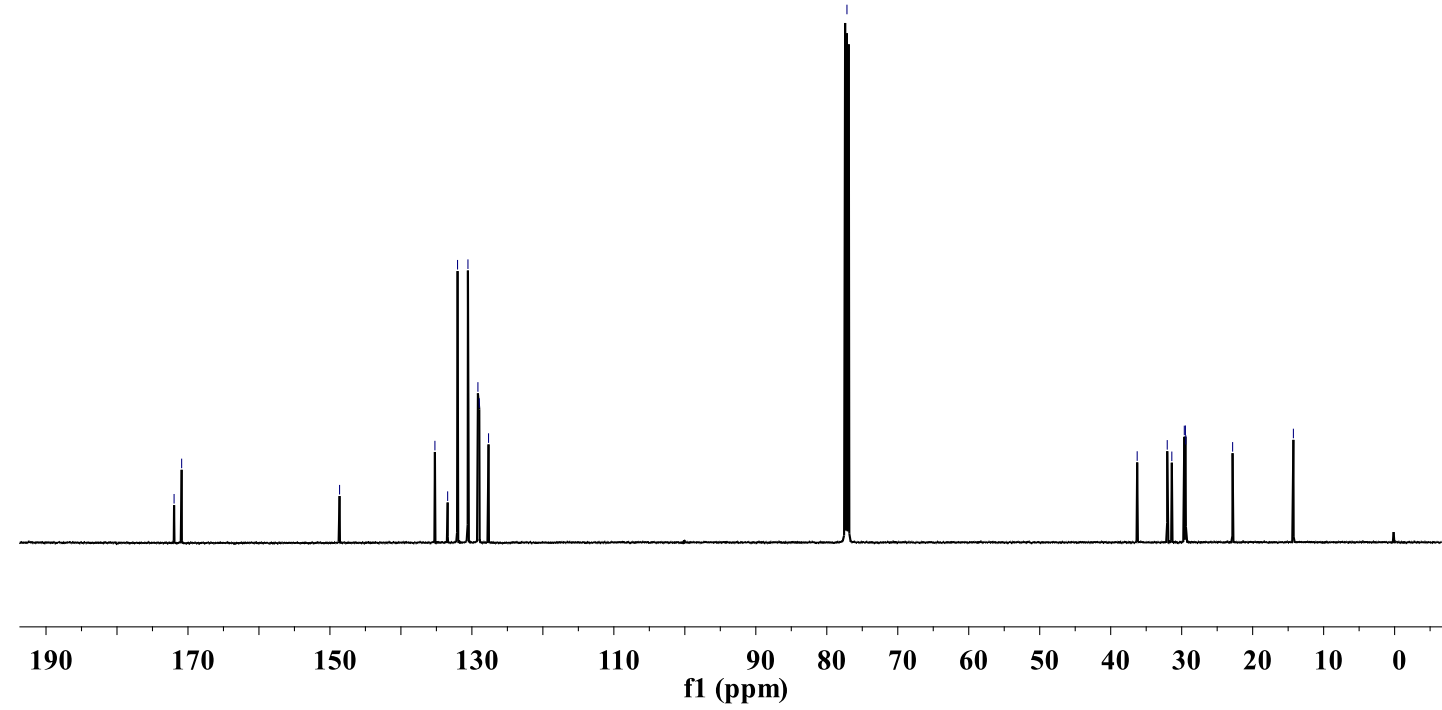

Figure S11. ${ }^{1} \mathrm{H}$ and ${ }^{13} \mathrm{C}$ NMR spectra of M3. 


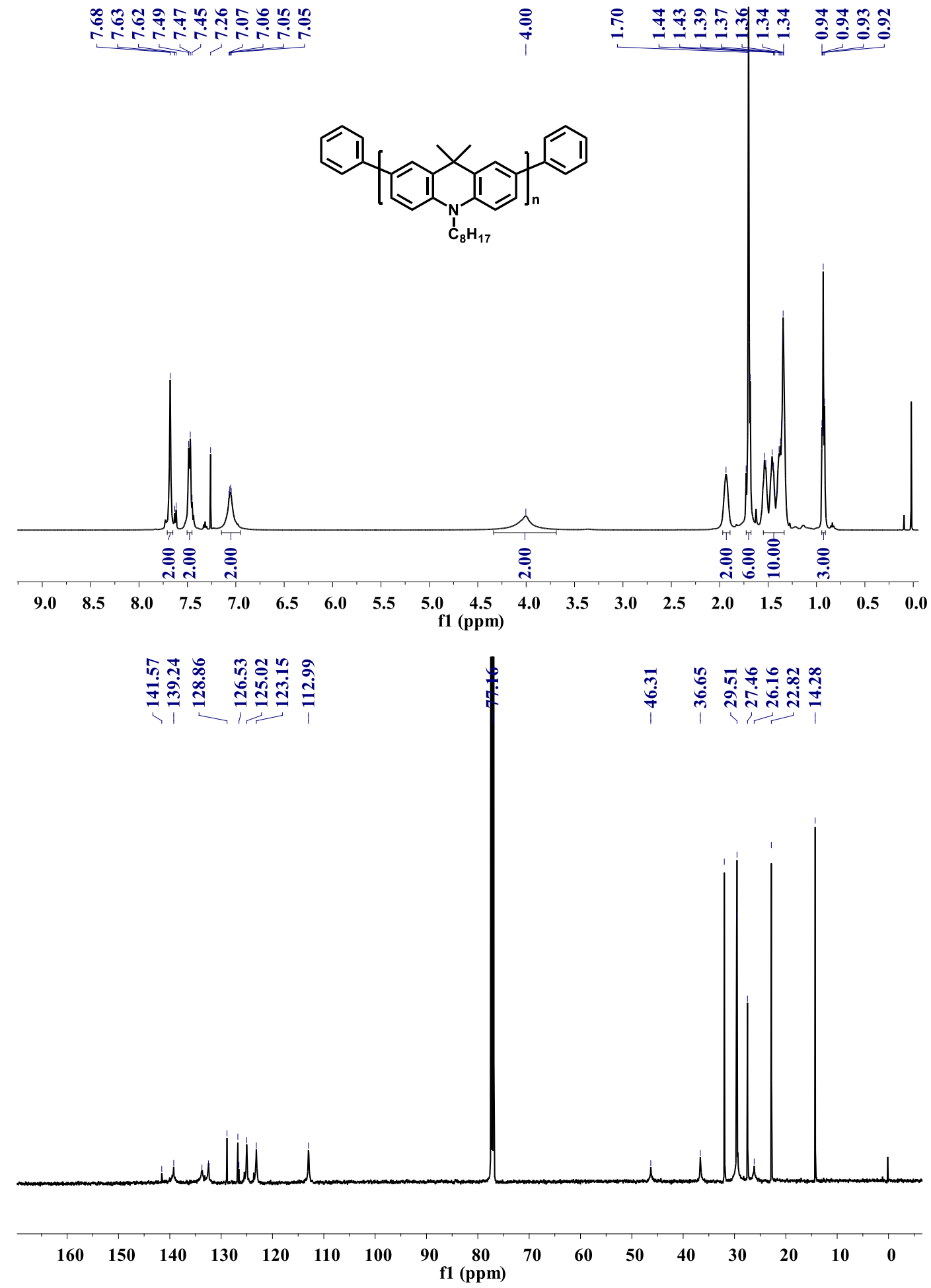

Figure S12. ${ }^{1} \mathrm{H}$ and ${ }^{13} \mathrm{C}$ NMR spectra of Homo-PAc. 

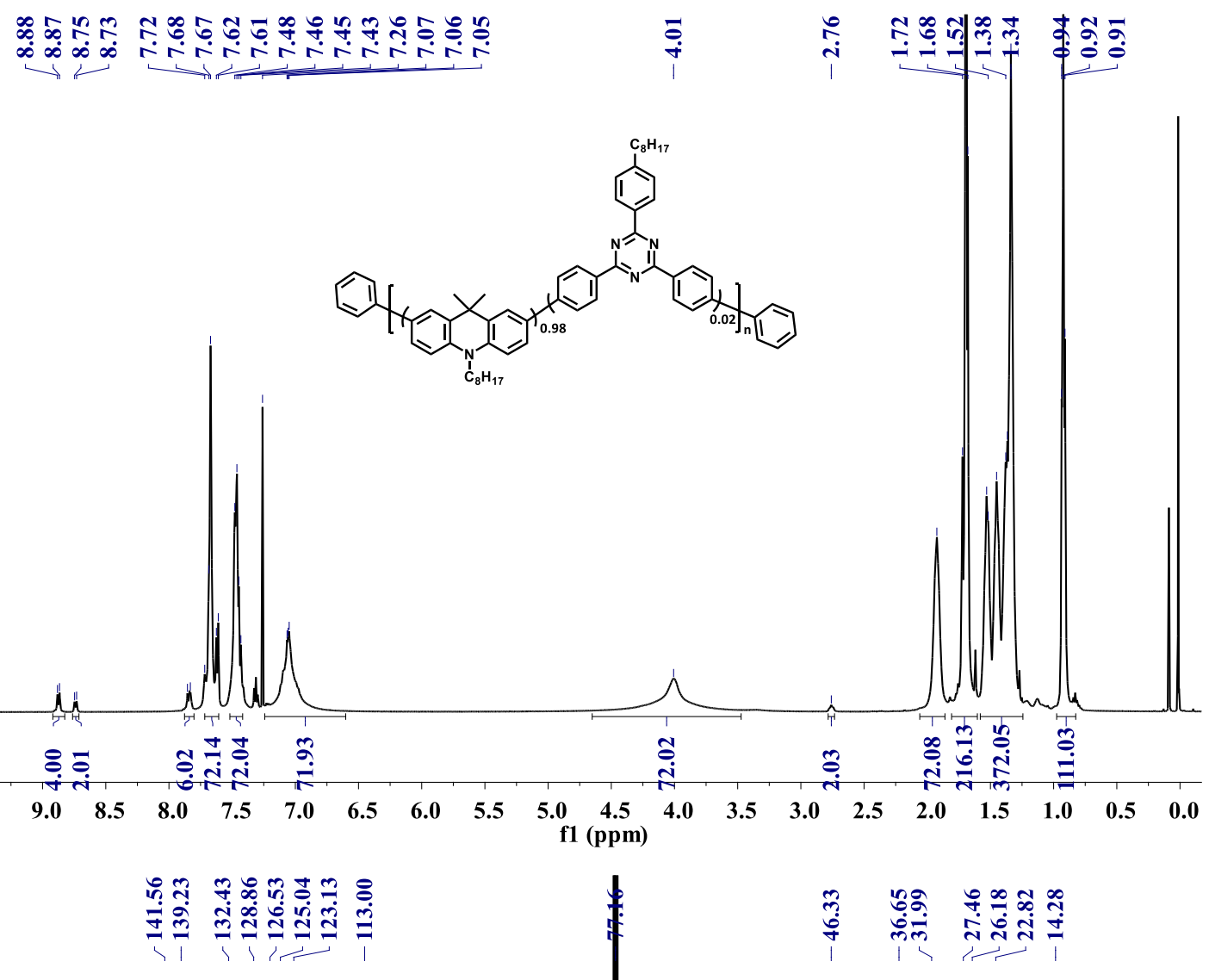

Figure S13. ${ }^{1} \mathrm{H}$ and ${ }^{13} \mathrm{C}$ NMR spectra of Ran-P(Ac-TRZ02). 


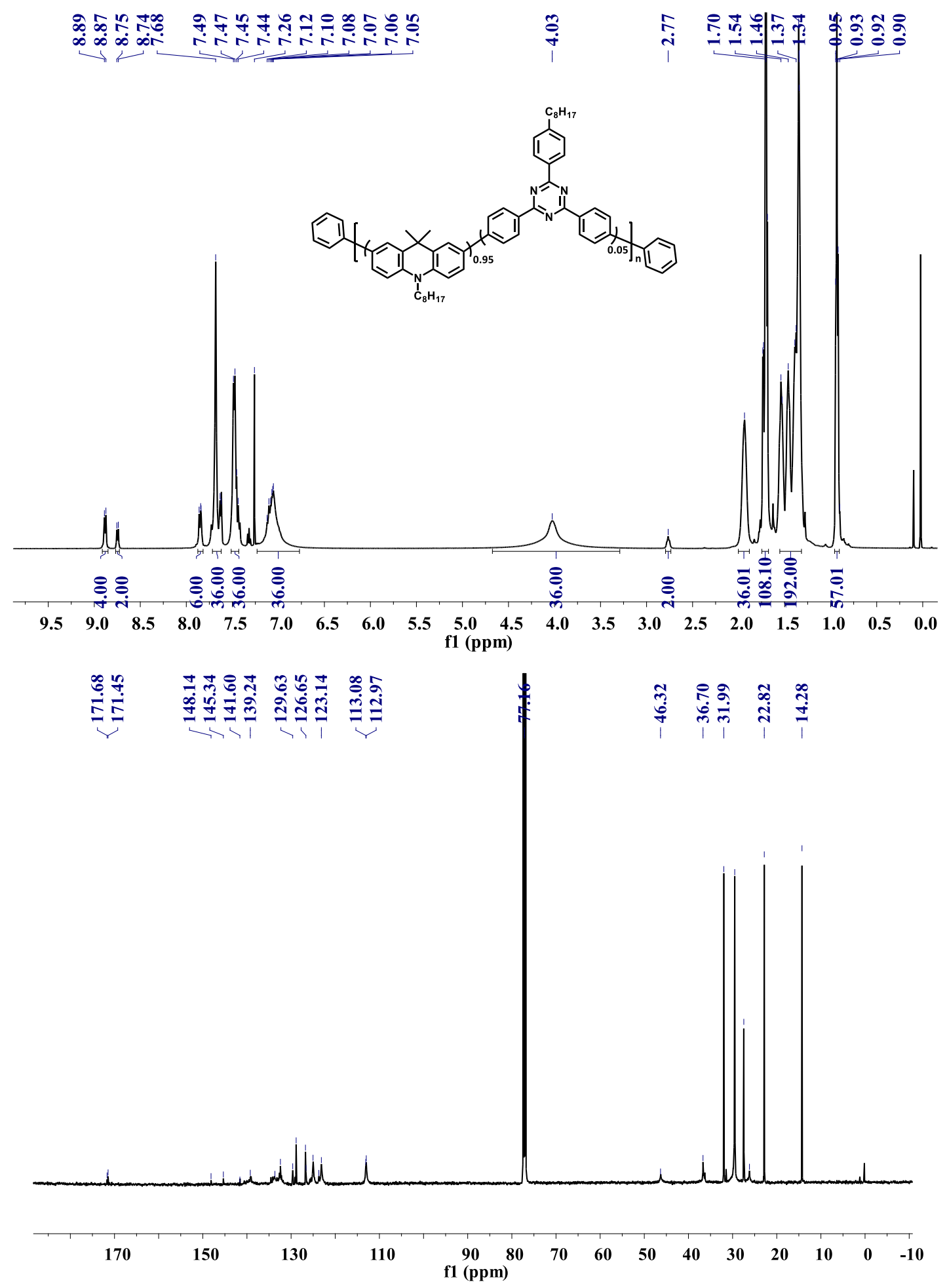

Figure S14. ${ }^{1} \mathrm{H}$ and ${ }^{13} \mathrm{C}$ NMR spectra of Ran-P(Ac-TRZ05). 

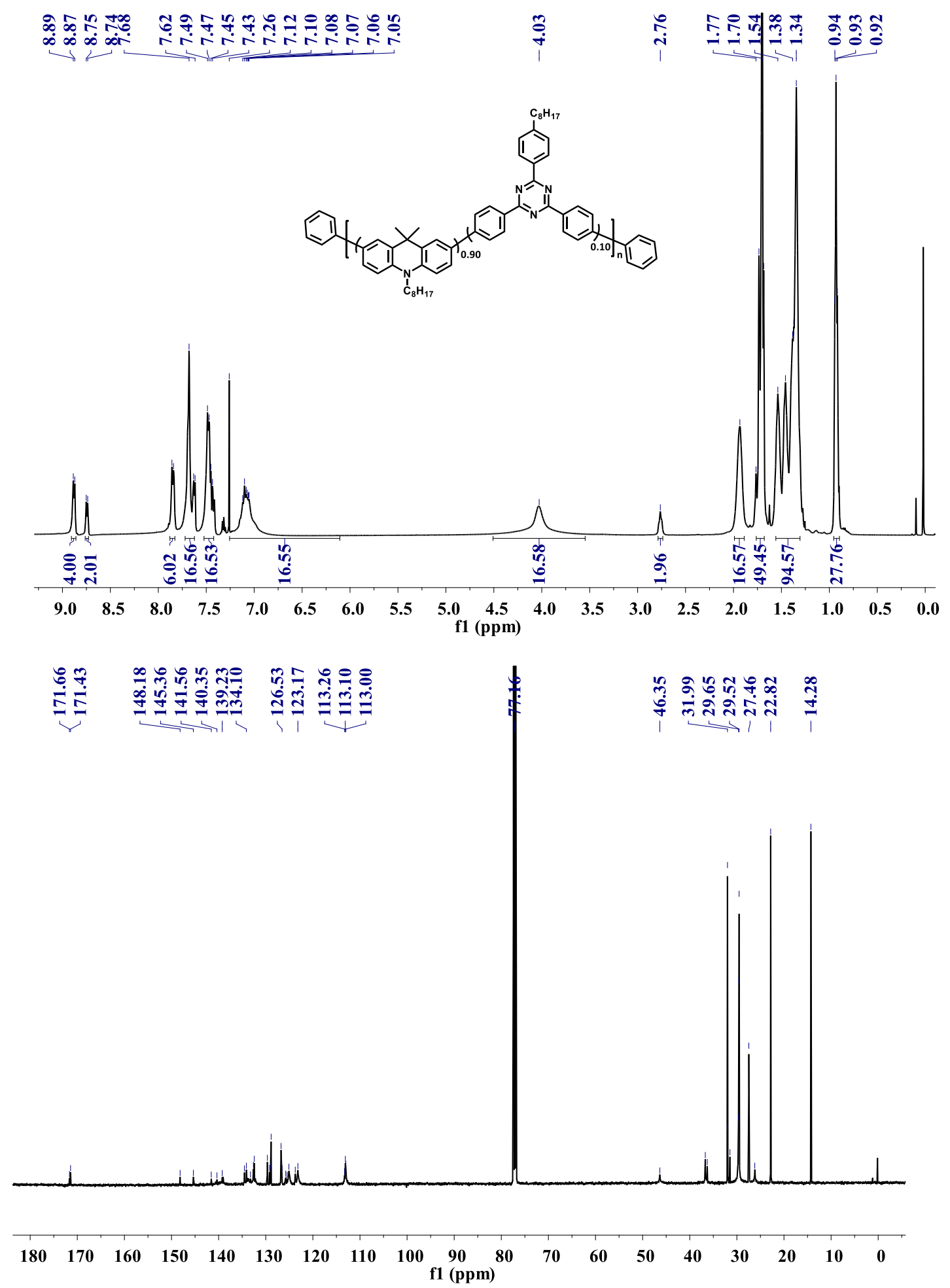

Figure S15. ${ }^{1} \mathrm{H}$ and ${ }^{13} \mathrm{C}$ NMR spectra of Ran-P(Ac-TRZ10). 

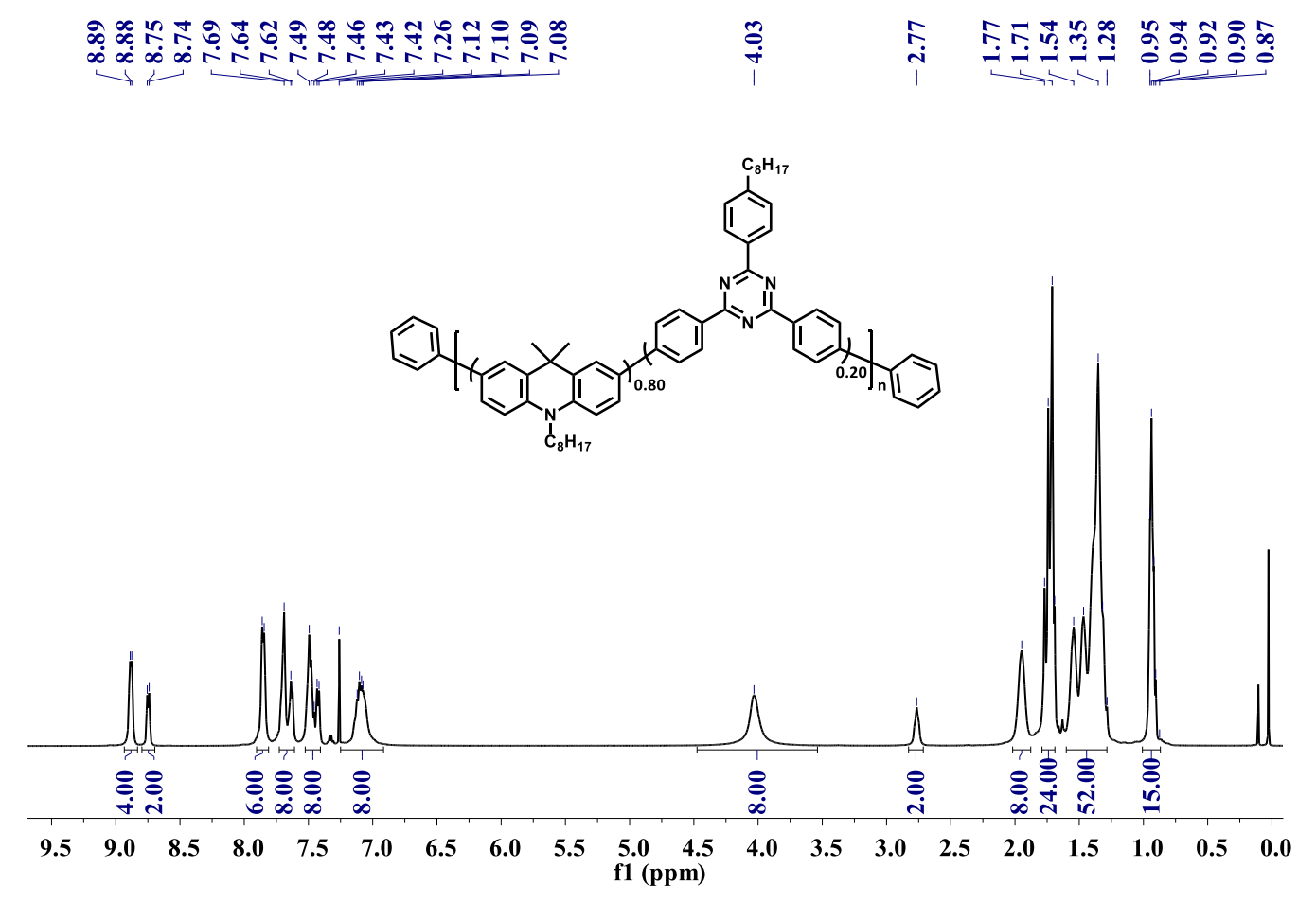

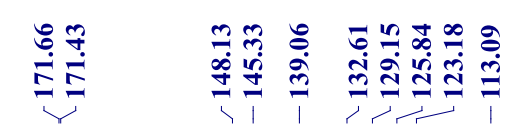

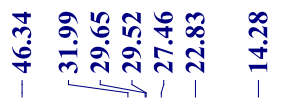

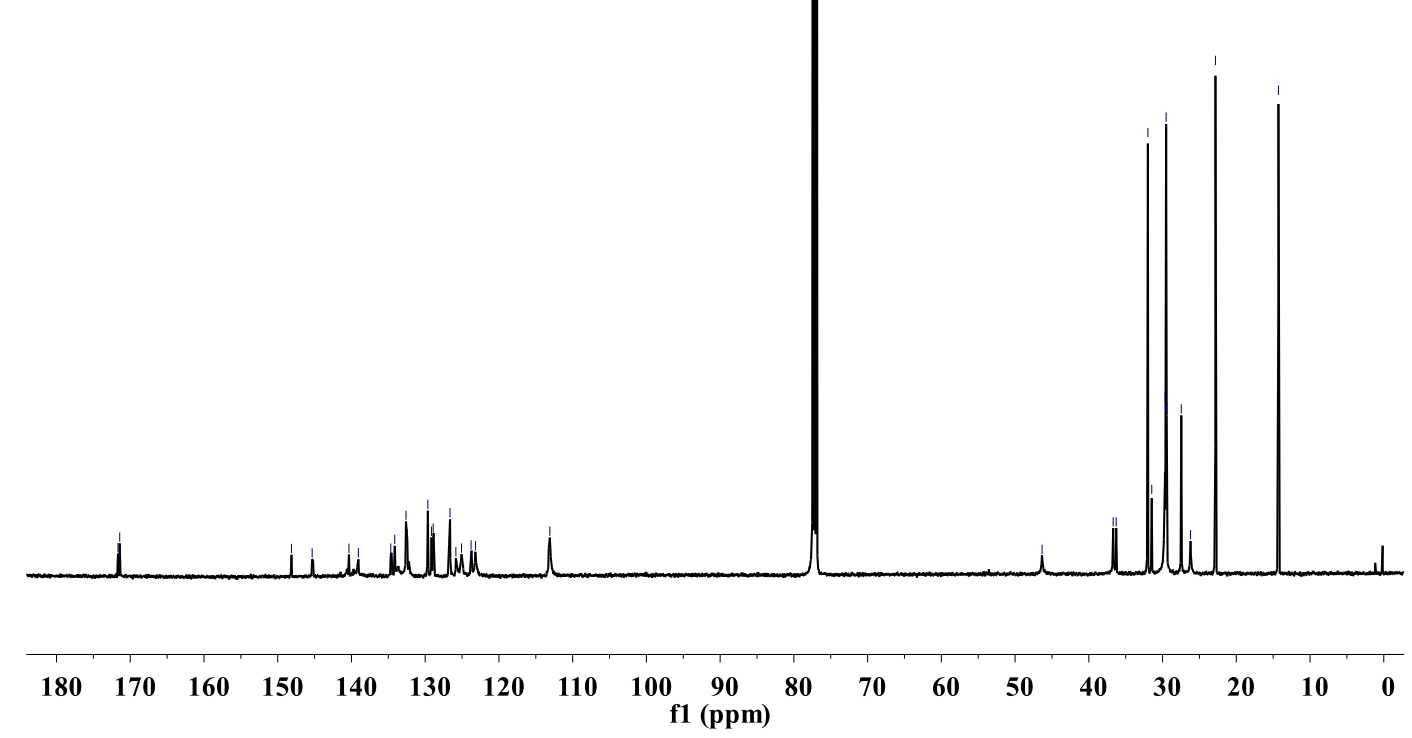

Figure S16. ${ }^{1} \mathrm{H}$ and ${ }^{13} \mathrm{C}$ NMR spectra of Ran-P(Ac-TRZ20). 


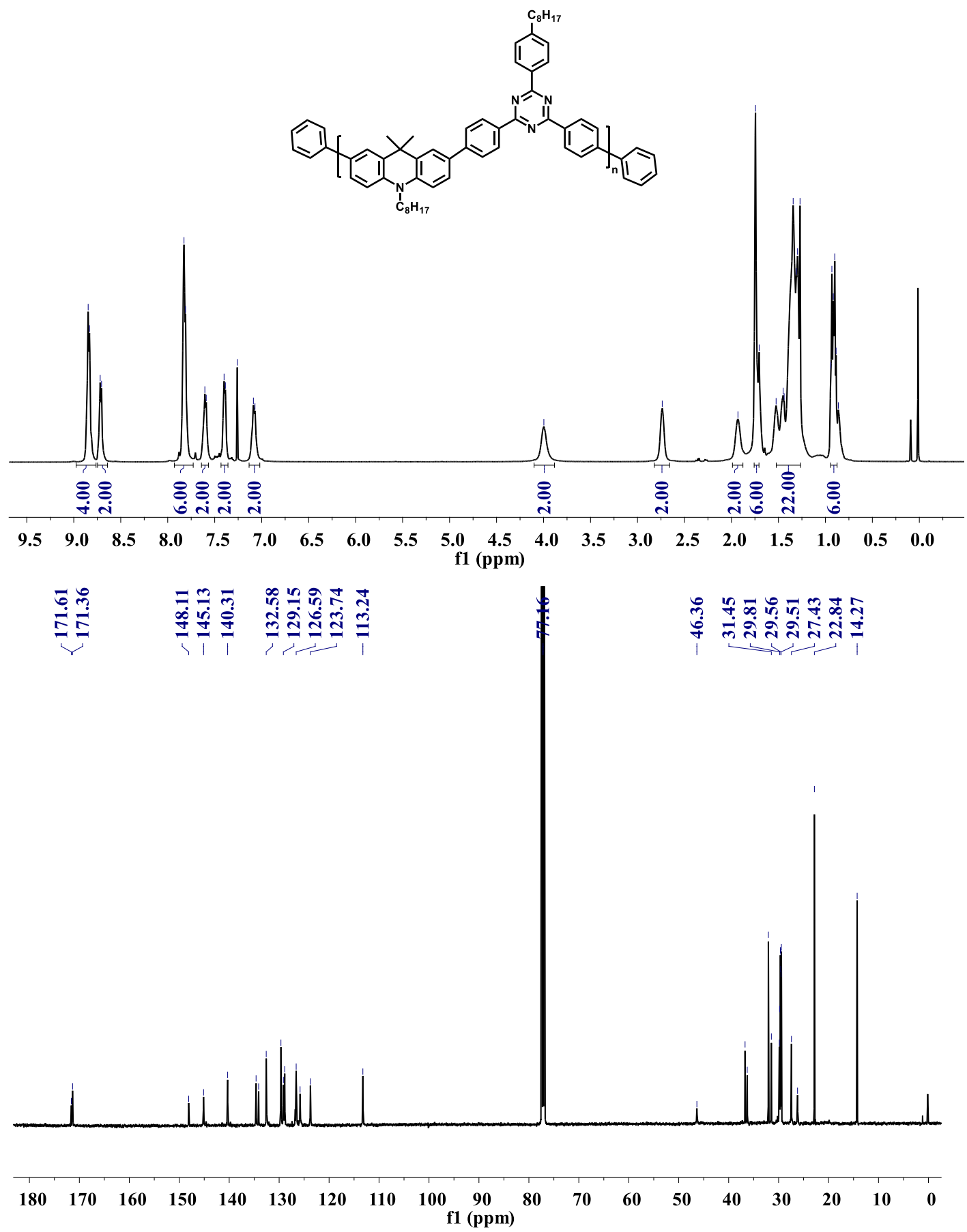

Figure S17. ${ }^{1} \mathrm{H}$ and ${ }^{13} \mathrm{C}$ NMR spectra of Alt-P(Ac-TRZ). 

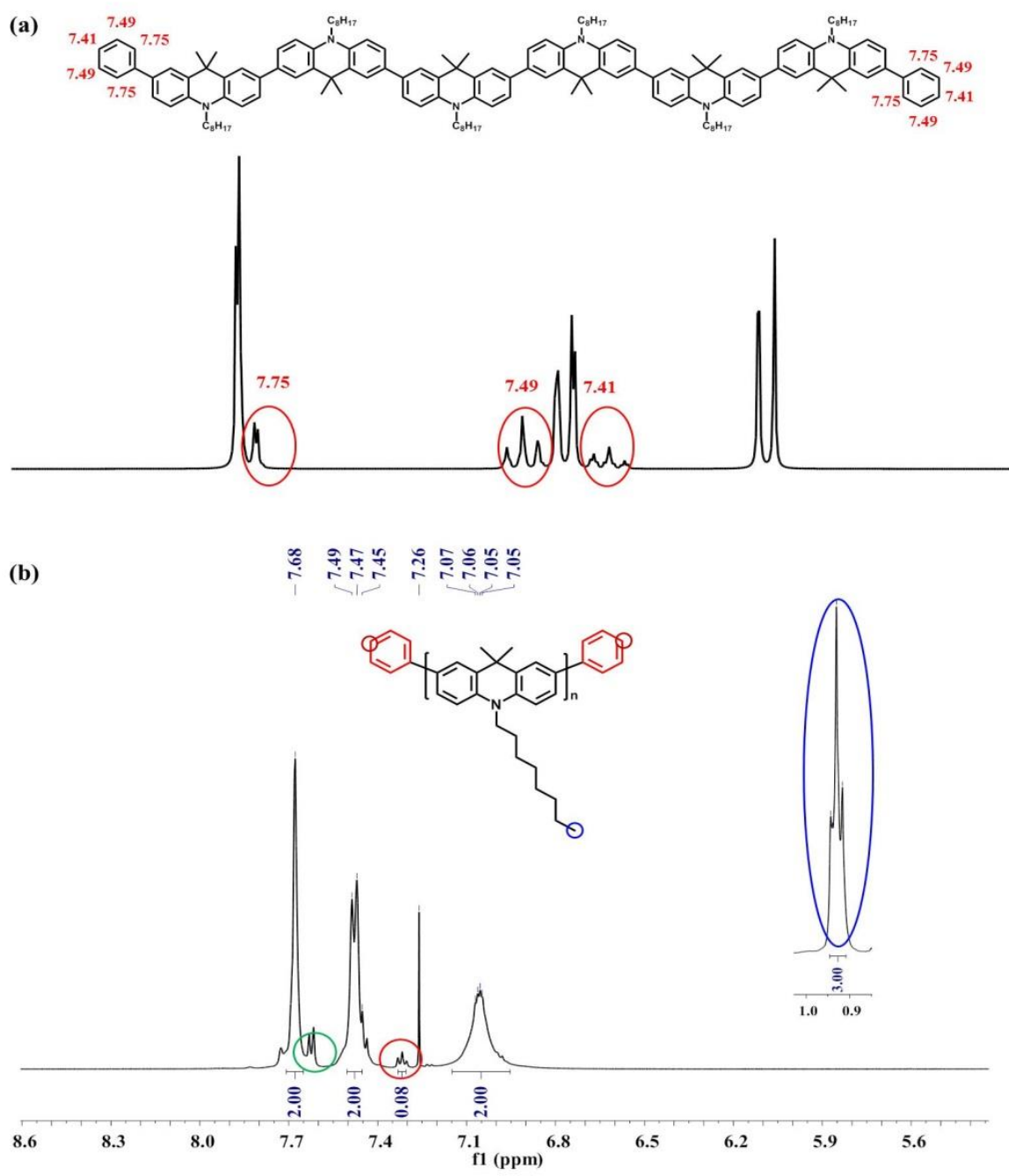

Figure S18. Comparison of ${ }^{1} \mathrm{H}$ NMR spectra between six Ac repeating units end-capped by phenyl (from simulation using ChemDraw 18.0 with $\mathrm{CDCl}_{3}$ as the solvent) and Homo-PAc (from experiment).

Taking Homo-PAc as an example, we have compared the simulated and experimental-obtained ${ }^{1} \mathrm{H}$ NMR spectra. As one can see, two additional signals are clearly discernible, and match well with those of the end-capped phenyl group (Figure 18b): the doublet one at $7.6 \mathrm{ppm}$ (in green circle) is from the ortho-position $\mathrm{H}$, the other triplet one at $7.3 \mathrm{ppm}$ (in red circle) can be reasonable ascribed to the para-position $\mathrm{H}$. And the signal from the meta-position $\mathrm{H}$ may overlap with those of the polymeric backbone. 
On the other hand, a characteristic signal can be found at about $0.9 \mathrm{ppm}$, which originates from the methyl tail in the octyl side chain (in blue circle). By comparing their integral ratio between two signals at $0.9 \mathrm{ppm}$ and $7.3 \mathrm{ppm}$, the number of repeating units in Homo-PAc is calculated to be 25 . That is, the molecular weight of Homo-PAc is $8.14 \mathrm{kDa}$, close to the value determined by GPC $(9.85 \mathrm{kDa})$.

As for all the polymers, the $7.3 \mathrm{ppm}$ signal, corresponding to the para-position $\mathrm{H}$ in the end-capped phenyl group, is found to be distinguished from the whole ${ }^{1} \mathrm{H}$ NMR spectra. So their molecular weights can be estimated with the same procedure, and the data are listed in the followed table. The agreement with the GPC values further verifies the reasonability of the $7.3 \mathrm{ppm}$ origination.

\begin{tabular}{ccc}
\hline & \multicolumn{2}{c}{$\mathrm{M}_{\mathrm{n}}[\mathrm{kDa}]$} \\
\cline { 2 - 3 } Polymers & From GPC & From ${ }^{1} \mathrm{H}$ NMR \\
\hline Homo-PAc & 9.85 & 8.14 \\
Ran-P(Ac-TRZ02) & 7.53 & 6.39 \\
Ran-P(Ac-TRZ05) & 9.36 & 8.83 \\
Ran-P(Ac-TRZ10) & 9.23 & 8.36 \\
Ran-P(Ac-TRZ20) & 13.76 & 12.82 \\
Alt-P(Ac-TRZ) & 5.91 & 5.84 \\
\hline
\end{tabular}


Table S1. Physical properties of the polymers.

\begin{tabular}{|c|c|c|c|c|c|c|}
\hline \multirow{2}{*}{ Polymers } & \multicolumn{2}{|c|}{ TRZ in the polymers [mol \%] } & \multirow{2}{*}{$\begin{array}{c}\mathrm{M}_{\mathrm{n}}{ }^{\mathrm{b}} \\
{[\mathrm{kDa}]}\end{array}$} & \multirow{2}{*}{$\mathrm{PDI}^{\mathrm{b}}$} & \multirow{2}{*}{$\begin{array}{c}\mathrm{T}_{\mathrm{g}} \mathrm{c} \\
{\left[{ }^{\circ} \mathrm{C}\right]}\end{array}$} & \multirow{2}{*}{$\begin{array}{r}\mathrm{T}_{\mathrm{d}}^{\mathrm{d}} \\
{\left[{ }^{\circ} \mathrm{C}\right]}\end{array}$} \\
\hline & feed ratio & actual ratio $^{b}$ & & & & \\
\hline Homo-PAc & 0 & 0 & 9.85 & 2.19 & 118 & 385 \\
\hline Ran-P(Ac-TRZ02) & 2 & 2.7 & 7.53 & 2.18 & 114 & 386 \\
\hline Ran-P(Ac-TRZ05) & 5 & 5.3 & 9.36 & 2.09 & 118 & 391 \\
\hline Ran-P(Ac-TRZ10) & 10 & 10.8 & 9.23 & 1.93 & 123 & 389 \\
\hline Ran-P(Ac-TRZ20) & 20 & 20.0 & 13.76 & 2.11 & 137 & 395 \\
\hline Alt-P(Ac-TRZ) & 50 & 50.0 & 5.91 & 1.27 & 170 & 400 \\
\hline
\end{tabular}

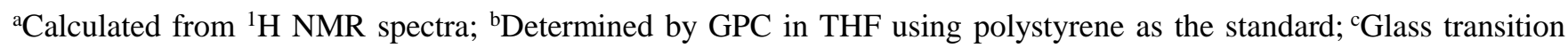
temperatures determined by DSC; ${ }^{\mathrm{d} D e c o m p o s i t i o n}$ temperatures (corresponding to a $5 \%$ weight loss) determined by TGA. 
Table S2. Summary of photophysical properties.

\begin{tabular}{cccccccccc}
\hline & $\tau_{\mathrm{p}}$ & $\tau_{\mathrm{d}}$ & $\Phi_{\mathrm{p}} / \Phi_{\mathrm{d}} / \Phi_{\mathrm{PL}}$ & $k_{\mathrm{p}}$ & $k_{\mathrm{d}}$ & $k_{\mathrm{r}}^{S}$ & $k_{\mathrm{ISC}}$ & $k_{\mathrm{RISC}}$ & $\mathrm{k}_{\mathrm{nr}}^{\mathrm{T}}$ \\
& {$[\mathrm{ns}]$} & {$[\mathrm{us}]$} & {$[\%]$} & {$\left[10^{7} \mathrm{~s}^{-1}\right]$} & {$\left[10^{5} \mathrm{~s}^{-1}\right]$} & {$\left[10^{7} \mathrm{~s}^{-1}\right]$} & {$\left[10^{7} \mathrm{~s}^{-1}\right]$} & {$\left[10^{5} \mathrm{~s}^{-1}\right]$} & {$\left[10^{5} \mathrm{~s}^{-1}\right]$} \\
\hline Homo-PAc & 2.8 & -- & $30 /--/ 30$ & 35.71 & -- & 10.71 & 25.00 & -- & -- \\
Ran-P(Ac-TRZ02) & 48.5 & 5.48 & $8.7 / 2.3 / 11$ & 2.06 & 1.82 & 0.18 & 1.88 & 0.53 & 1.77 \\
Ran-P(Ac-TRZ05) & 48.2 & 5.13 & $7.4 / 1.6 / 9$ & 2.07 & 1.95 & 0.15 & 1.92 & 0.45 & 1.81 \\
Ran-P(Ac-TRZ10) & 51.8 & 3.98 & $7.9 / 1.1 / 9$ & 1.93 & 2.51 & 0.15 & 1.78 & 0.38 & 2.47 \\
Ran-P(Ac-TRZ20) & 44.4 & 1.86 & $6.8 / 0.2 / 7$ & 2.25 & 5.38 & 0.15 & 2.10 & 0.17 & 5.37 \\
Alt-P(Ac-TRZ) & 13.3 & -- & $24 /--/ 24$ & 7.52 & -- & 1.80 & 5.72 & - & - \\
\hline
\end{tabular}

Notes: The data is collected in neat films for the polymers under nitrogen atmosphere. $\tau_{\mathrm{p}}$ and $\tau_{\mathrm{d}}$ are prompt and delayed lifetimes; $\Phi_{\mathrm{PL}}$ is the total PLQY; $\Phi_{\mathrm{p}}$ and $\Phi_{\mathrm{d}}$ are the PLQY of prompt and delayed components; $k_{\mathrm{p}}, k_{\mathrm{d}}, k_{\mathrm{r}}^{\mathrm{S}}, k_{\mathrm{ISC}}, k_{\mathrm{RISC}}$ and $k_{\mathrm{nr}}{ }^{\mathrm{T}}$ are the rate constants of the prompt fluorescence, delayed fluorescence, singlet radiation, intersystem crossing from $S_{1}$ to $T_{1}$, reverse intersystem crossing from $T_{1}$ to $S_{1}$ and triplet non-radiation, respectively. All these parameters are obtained following the three-energy-level model by ignoring the non-radiative pathway of $S_{1}$ and the radiation of $T_{1}$.

$k_{\mathrm{p}}=1 / \tau_{\mathrm{p}} \quad k_{\mathrm{d}}=1 / \tau_{\mathrm{d}} \quad k_{\mathrm{r}}^{\mathrm{S}}=k_{\mathrm{p}} \cdot \Phi_{\mathrm{p}} \quad k_{\mathrm{ISC}}=k_{\mathrm{p}} \cdot\left(1-\Phi_{\mathrm{p}}\right) \quad k_{\mathrm{RISC}}=\left(k_{\mathrm{p}} \cdot k_{\mathrm{d}} / k_{\mathrm{ISC}}\right) \cdot\left(\Phi_{\mathrm{d}} / \Phi_{\mathrm{p}}\right) \quad k_{\mathrm{nr}}{ }^{\mathrm{T}}=k_{\mathrm{d}}-k_{\mathrm{RISC}} \cdot \Phi_{\mathrm{p}}$ 
Table S3. Summary of photophysical properties of the polymers in $5 \mathrm{wt} . \%$ doped mCP films.

\begin{tabular}{cccc}
\hline Polymer & $\begin{array}{c}\lambda_{\mathrm{PL}}{ }^{\mathrm{a}} \\
{[\mathrm{nm}]}\end{array}$ & $\begin{array}{c}\mathrm{S}_{1} / \mathrm{T}_{1} / \Delta \mathrm{E}_{\mathrm{ST}}{ }^{\mathrm{b}} \\
{[\mathrm{eV}]}\end{array}$ & $\begin{array}{c}\tau_{\mathrm{p}} / \tau_{\mathrm{d}}{ }^{\mathrm{c}} \\
{[\mathrm{ns} / \mathrm{us}]}\end{array}$ \\
\hline Homo-PAc & 419 & $3.10 / 2.48 / 0.62$ & $7.2 /$ n.d. \\
Ran-P(Ac-TRZ02) & 499 & $2.79 / 2.44 / 0.35$ & $35.2 / 4.69$ \\
Ran-P(Ac-TRZ05) & 504 & $2.75 / 2.41 / 0.34$ & $30.2 / 4.13$ \\
Ran-P(Ac-TRZ10) & 510 & $2.71 / 2.36 / 0.35$ & $33.2 / 3.68$ \\
Ran-P(Ac-TRZ20) & 510 & $2.70 / 2.34 / 0.36$ & $36.2 / 1.61$ \\
Alt-P(Ac-TRZ) & 488 & & $12.3 / \mathrm{n} . \mathrm{d}$. \\
\hline
\end{tabular}

${ }^{\text {a }}$ Fluorescence measured in 5 wt. $\%$ doped mCP films. ${ }^{\text {b }} \mathrm{S}_{1}$ and $\mathrm{T}_{1}$ are estimated by onsets of fluorescence and phosphorescence in $5 \mathrm{wt} . \%$ doped $\mathrm{mCP}$ films respectively and $\Delta \mathrm{E}_{\mathrm{ST}}=\mathrm{S}_{1}-\mathrm{T}_{1} \cdot{ }^{\mathrm{c}}$ Prompt and delayed PL transient decay lifetimes obtained in $5 \mathrm{wt} . \%$ doped $\mathrm{mCP}$ films under nitrogen atmosphere with excitation of $375 \mathrm{~nm}$. 
Table S4. Computational results.

\begin{tabular}{cccccccc}
\hline Chain segments & $\begin{array}{c}\mathrm{HOMO}^{\mathrm{a}} \\
{[\mathrm{eV}]}\end{array}$ & $\begin{array}{c}\mathrm{LUMO}^{\mathrm{a}} \\
{[\mathrm{eV}]}\end{array}$ & $\begin{array}{c}\mathrm{E}_{\mathrm{g}}{ }^{\mathrm{a}} \\
{[\mathrm{eV}]}\end{array}$ & $\begin{array}{c}\mathrm{E}_{\mathrm{VA}}\left(\mathrm{S}_{1}\right)^{\mathrm{b}} \\
{[\mathrm{eV}]}\end{array}$ & $\begin{array}{c}\mathrm{E}_{\mathrm{VA}}\left(\mathrm{T}_{1}\right)^{\mathrm{b}} \\
{[\mathrm{eV}]}\end{array}$ & $\begin{array}{c}\Delta \mathrm{E}_{\mathrm{ST}}{ }^{\mathrm{b}} \\
{[\mathrm{eV}]}\end{array}$ & $f\left(\mathrm{~S}_{1}\right)^{\mathrm{b}}$ \\
\hline D-A-D-A-D-A & -5.06 & -1.82 & 3.24 & 2.8768 & 2.5329 & 0.3439 & 1.7254 \\
D-D-D-A-D-D-D & -4.64 & -1.70 & 2.94 & 2.7212 & 2.5052 & 0.2160 & 0.4915 \\
D-D-D-D-D-D & -4.60 & -0.61 & 3.99 & 3.4959 & 2.8626 & 0.6333 & 3.5243 \\
\hline
\end{tabular}

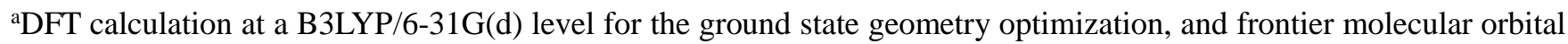
information; ${ }^{b} \mathrm{TD}-\mathrm{DFT}$ calculation to predict energies of the lowest excited states $\left(\mathrm{S}_{1}\right.$ and $\left.\mathrm{T}_{1}, \Delta \mathrm{E}_{\mathrm{ST}}=\mathrm{S}_{1^{-}} \mathrm{T}_{1}\right)$ and the oscillator strength $(f)$ of $S_{1}$, regardless of the difference of charge transfer degree. 